\title{
Relaxation structurale et liquides vitrifiables
}

\author{
C. Alba-Simionesco ${ }^{1}$, V. Krakoviack ${ }^{1,2}$ et A. Tölle ${ }^{3}$ \\ ${ }^{1}$ Laboratoire de Chimie Physique, UMR 8000 du CNRS, bâtiment 349, Université Paris-Sud, \\ 91405 Orsay, France \\ ${ }^{2}$ Laboratoire de Chimie Théorique et des Matériaux Hybrides, ENS Lyon, 46 allée d'ltalie, \\ 69364 Lyon cedex 07, France \\ ${ }^{3}$ Deloitte Consulting, Mühlemattstrasse 22, 4104 Oberwil, Suisse
}

\section{INTRODUCTION : VISCOSITE ET RELAXATION STRUCTURALE}

Une méthode classique pour suivre l'évolution de l'écoulement ou de la fluidité d'un liquide consiste à mesurer l'augmentation de sa viscosité de cisaillement $\eta$ lorsqu'on abaisse la température dans des conditions normales de pression (1atm). Le résultat est représenté traditionnellement par la courbe $\log \eta=\mathrm{f}(1 / \mathrm{T})$ dans un diagramme d'Arrhénius, et ajusté numériquement par une expression de type

$$
\eta(T)=\eta_{\infty} \exp [E(T) / T]
$$

où $\mathrm{E}(\mathrm{T})$ est une barrière d'activation effective. Les unités de $\mathrm{E}$ sont en degrés $\mathrm{K}$.

Bien que la viscosité soit un coefficient de transport, (une grandeur macroscopique obtenue à fréquence nulle), on peut la relier par la théorie de la viscoélasticité de Maxwell (1863) à l'évolution du temps moyen de relaxation structurale $\tau_{\alpha}$ par la relation

$$
\eta=\mathrm{G}_{\infty} \cdot \tau_{\alpha}
$$

où $\mathrm{G}_{\infty}$ est le module de cisaillement à fréquence infinie (de l'ordre de quelques GPa pour un liquide visqueux).

On appelle relaxation structurale ou bien encore $\alpha$ ou principale (Litovitz 1965), -ce temps caractéristique associé aux processus de réorganisation de la structure du liquide, c'est-àdire au temps nécessaire au système pour se réajuster structuralement après un changement de l'ordre local et à moyenne portée. C'est un aspect tout à fait typique de la dynamique des liquides à des températures autour du point de fusion et en dessous.

Ce temps de relaxation est mesuré par diverses techniques spectroscopiques, chacune sensible à la dynamique d'une variable spécifique, et sa valeur peut changer d'un ordre de grandeur d'une sonde à l'autre. Il désigne le retour à l'équilibre d'une grandeur $\mathrm{A}$ à $\mathrm{A}_{\mathrm{eq}}$, après l'application d'une petite perturbation, (ici de température $\Delta \mathrm{T}$ ), dont la fonction de relaxation peut s'écrire sous la forme $\phi(t)$ ci-dessous. Ce retour à l'équilibre peut s'observer dans le temps ou en fréquence si l'on applique une contrainte sinusoïdale. La réponse du système s'exprime sous la forme d'une susceptibilité complexe reliée à la fonction de relaxation $\phi(t)$ par :

$$
\chi(\omega)=\chi_{\infty}+\left(\chi_{\infty}-\chi_{0}\right) \int^{\infty} d t e^{i \omega t} \phi(t)
$$


Par la théorie de la réponse linéaire, on peut relier la fonction de relaxation d'une grandeur vers l'équilibre d'un système à la fonction de corrélation des fluctuations spontanées de cette grandeur autour de sa valeur d'équilibre.

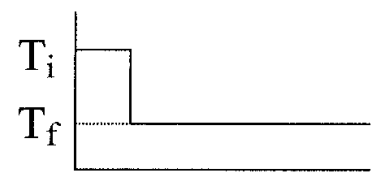

Figure 1: évolution temporelle d'une fonction de relaxation $\phi(t)$ de la grandeur $\mathrm{A}$, après une perturbation $\Delta \mathrm{T}$

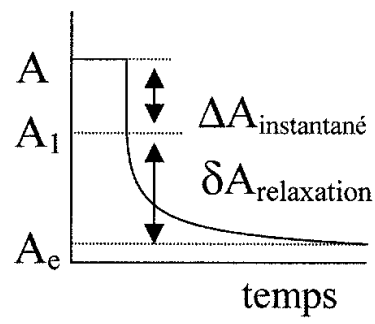

Le temps de relaxation (ou la fréquence associée) varie considérablement avec la température : il augmente de plusieurs décades pour un refroidissement de quelques dizaines de Kelvins. C'est cette variation qui est à l'origine du phénomène de transition vitreuse, illustré sur la figure 2 .

\section{PHENOMENOLOGIE DES LIQUIDES SURFONDUS.}

\section{1 comportement super-Arrhénius du temps de relaxation}

Lorsqu'un liquide peut être refroidi ou comprimé en évitant sa cristallisation, il finit par former un solide amorphe, appelé verre. Le changement d'état observé est dû à l'entrée et au maintien du système hors d'équilibre (en termes techniques à une rupture opératoire d'ergodicité), le temps de relaxation moyen nécessaire aux réarrangements structuraux devenant de l'ordre de grandeur ou supérieur au temps caractéristique de la mesure expérimentale. Selon la technique expérimentale et l'histoire thermique considérées, on observera un domaine de transition vitreuse plus ou moins large. Pour définir alors cette température de gel de la relaxation structurale, il faut convenir d'une vitesse de refroidissement ou de surcompression par rapport au temps caractéristique de mesure expérimentale. Il apparaît clairement d'après cette définition que la transition vitreuse telle qu'elle est observée n'est pas un phénomène thermodynamique mais est ad hoc à la nature et à la cinétique de l'expérience destinée à la mettre en évidence. Il ne s'agit pas là à proprement parler d'une transition de phase, mais d'un gel de certains degrés de liberté à l'échelle du temps de mesure expérimental ; ce gel se manifeste par un saut, d'amplitude variable d'un liquide à l'autre, des coefficients thermodynamiques comme la chaleur spécifique $\mathrm{C}_{\mathrm{P}}$, sans qu'il y ait de modification structurale appréciable, et par une rupture de pente dans la mesure de la viscosité fonction de la température (cf. Fig.2).La température Tg et la pression Pg sont en 
général définies comme les limites macroscopiques d'équilibre de la phase liquide métastable et correspondent à un temps de relaxation de 100 à $1000 \mathrm{sec}$, ou une valeur de la viscosité de l'ordre de $10^{13}$ poise $\left(10^{14} \mathrm{~Pa}^{1} \mathrm{~s}^{-1}\right)$.

Une des caractéristiques les plus remarquables de la plupart des liquides approchant la transition vitreuse est l'augmentation considérable de la viscosité et du temps moyen de relaxation structurale : on observe une variation sur 15 décades ou plus pour un simple changement de température d'un facteur 2 et on a souvent recours à une équation empirique dite de Vogel (1921)-Tammann(1926)-Fulcher(1925) pour représenter la courbure observée dans le graphe (cf. Figure 2).

$$
\log \eta=A+B /(T-T o)
$$

$\rho \mathrm{g} / \mathrm{cm}^{3}$

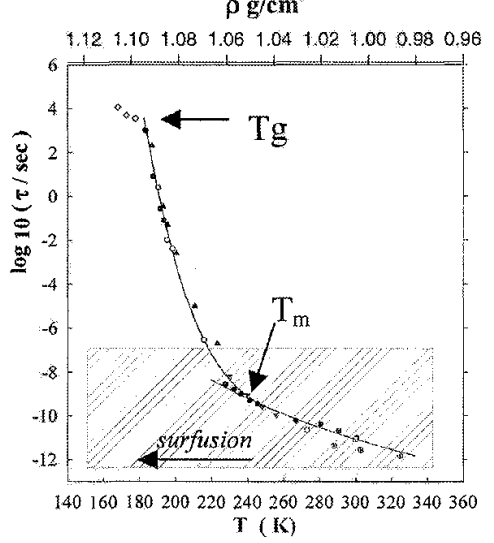

Figure 2 : évolution du temps de relaxation de la $\mathrm{m}$-toluidine avec la température à la pression atmosphérique combinant diverses techniques de spectroscopie. Les flèches indiquent la transition vitreuse et le point de fusion. Pour indication, la variation de la densité dans ces conditions est représentée en haut du graphe. La zone achurée délimite le domaine d'étude par diffusion de neutrons

Les propriétés dynamiques qui varient de manière spectaculaire avec la température sont interprétées habituellement en terme de cinétique thermiquement activée, impliquant le passage sous 1'effet de fluctuations thermiques d'une barrière d'énergie séparant deux minima d'énergie. La spécificité des phénomènes de transport et de relaxation dans les liquides vitrifiables tient au fait que les énergies apparentes d'activation, au lieu d'être constantes, augmentent lorsque la température diminue. Ceci est vrai pour la majorité des liquides vitrifiables qui sont alors dits 'fragiles' et 'intermédiaires' selon une classification introduite par C.A. Angell (1985) et regroupés sur une même figure en recalant leur $\mathrm{Tg}$. Les systèmes fragiles présentés sur la figure 3 sont des liquides moléculaires aromatiques, sur la base du benzène et de ses dérivés substitués, toluène, $\mathrm{m}$-toluidine et o-terphenyl, et un polymère, le poly(vinyl methyl ether).

L'utilité d'une description des liquides surfondus à base de dynamique thermiquement activée peut cependant dépendre du domaine de température et de pression (ou densité) considéré. Il est généralement accepté que les processus activés dominent le régime au voisinage de la transition des liquides vitrifiables dits 'fragiles' à la pression atmosphérique, pour lequel les énergies apparentes d'activation varient de $5 \mathrm{kT}$ jusqu'à $40 \mathrm{kT}$; il est accepté également qu'ils dominent la dynamique à toute température des systèmes dits 'forts' qui forment des réseaux tridimensionnels et pour lesquels les énergies nécessaires à briser les liaisons entre 'molécules' sont élevées ; c'est le cas de $\mathrm{SiO}_{2}$ ou de $\mathrm{GeO}_{2}$. 


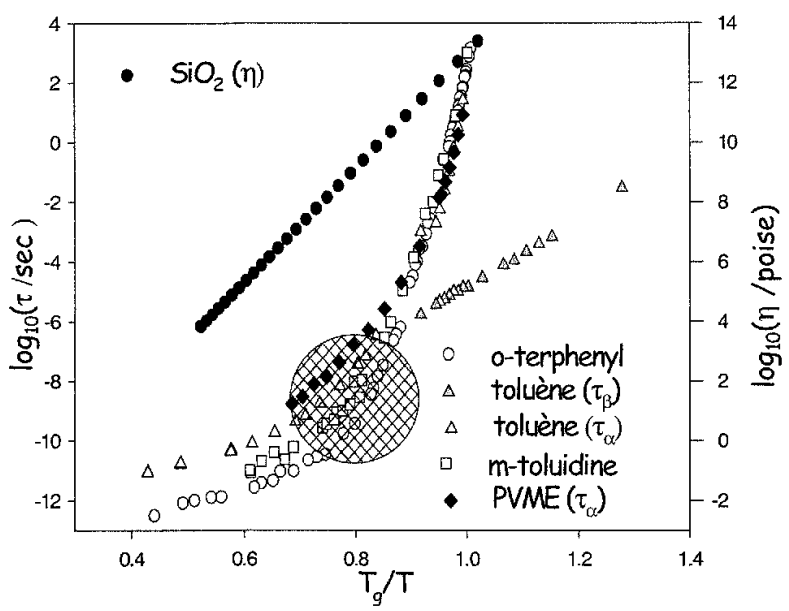

Figure 3 :diagramme arrhenien de la classification d'Angell, où des liquides de nature chimique très différentes sont représentés en normalisant l'axe des températures par leur $T_{g}$. La zone de recouvrement (ou cross-over) entre deux régimes dynamiques est achurée sur le graphe. Les données du toluène et du PVME ont été fournies par le Prof. Rössler et le Prof. Alegria.

Pour la plupart des liquides surfondus, il existe une température de 'cross-over', $\mathrm{T}^{*}$ ou Tc, à laquelle on observe le changement d'une dépendance en température approximativement Arrhénienne de la viscosité et des temps de relaxation à plus haute température à un comportement superArrhénien, croissant plus rapidement que la loi d'Arrhénius quand la température décroît. A partir de ce changement de régime, une série d'évènements expérimentaux se succède : ainsi se détache un processus de relaxation dite $\beta$ rapide, qui serait le précurseur de la relaxation $\alpha$, puis un processus de relaxation dit $\beta$ lent (ou secondaire ou Johari-Goldstein) visible dans de nombreux verres, ou encore les temps associés à la diffusion translationnelle et rotationnelle se séparent, enfin l'équation de Stokes-Einstein $(\eta=k T / 6 \pi \mathrm{aD}$ où $D$ est le coefficient de diffusion et a le diamètre de la molécule) n'est plus vérifiée.

L'augmentation de l'énergie d'activation s'accompagne d'une diminution de l'entropie du système. Dans de nombreux liquides cette diminution peut être notable (un facteur 2 à 4 du point de fusion $\mathrm{Tm}$ à $\mathrm{Tg}$ ) et l'extrapolation de ce comportement vers des températures plus basses que Tg (donc inaccessible pour le liquide) conduit au célèbre paradoxe de Kauzmann (1948): l'entropie du liquide métastable pourrait devenir inférieure à celle du cristal à une même température. Ce paradoxe, qui n'est, il faut insister là-dessus, qu'un résultat d'extrapolation, couplé avec l'apparente divergence de la viscosité et du temps de relaxation $\alpha_{\ell}$ (encore une fois, divergence extrapolée et non observée), suggère l'existence d'une transition de phase proprement thermodynamique vers un verre idéal à une température inférieure à $\mathrm{Tg}$, dont l'accès expérimental serait rendu impossible à cause des trop longs temps de relaxation. Cette transition vitreuse idéale a donné lieu à de nombreux modèles théoriques.

\subsection{Etirement de la fonction de relaxation.}

Il est classique dans l'étude de la dynamique des liquides surfondus d'observer des phénomènes de relaxation plus lents que la loi de décroissance exponentielle de Debye(1929) habituelle dans le cadre de l'étude de la matière condensée. Ainsi il ne sera pas rare que la régression d'une perturbation entre $90 \%$ et $10 \%$ de sa valeur initiale nécessite 2 décades de temps au lieu 1.34, ou bien, si le processus est observé dans en fonction de la fréquence, qu'un pic $\alpha$ soit d'une largeur à mi-hauteur de 2 décades plutôt que 1.14. Ce phénomène, découvert par 
Kohlrausch (1854), est appelé l'étirement de la relaxation structurale et en est la propriété la plus fondamentale. Il est très important du point de vue expérimental car il impose de disposer de moyens d'étude donnant accès à un domaine de fréquences de plusieurs décades. La loi de Kolhraush-Williams-Watt décroit dans le temps suivant une loi non-exponentielle, (il faut noter la confusion qu'introduit une notation malheureuse où l'exposant $\beta_{\mathrm{KW}}$ se rattache à la variation de la relaxation $\alpha$, et non de la relaxation plus rapide $\beta$ ou secondaire citée plus haut),

$$
\phi(\mathrm{t})=\tau_{\infty} \exp \left(-(t / \tau)^{\beta}{ }_{k W W}\right)
$$

où le temps moyen de relaxation peut être calculé comme suit :

$$
<\tau>=\int_{0}^{\infty} \phi(t) d t=\tau \beta^{-1} \Gamma\left(\beta^{-1}\right)
$$

Lorsque que la mesure est en fréquence, on se ramène à une loi empirique de ColeDavidson (1951):

$$
\chi^{*}=\chi_{\infty}+\frac{\chi_{s}-\chi_{\infty}}{\left(1+i \omega \tau_{c D}\right)^{\beta C D}}
$$

La correspondance des temps (KWW et $\mathrm{CD}$ ) et des exposants est bien développée dans Gomez et al. (2001).

La loi de puissance de von Schweidler(1907) s'applique plus spécifiquement aux premières étapes de la relaxation

$$
\phi(t)=f-h \cdot(t / \tau)^{b} \text { avec } \beta_{K W w} \text { et } b \text { compris entre } 0 \text { et } 1
$$

Cet exposant $\beta_{\mathrm{K} w w}$ généralement décroît avec la température et passe de valeurs voisines de 1 (exponentielle simple) à haute température et à des valeurs entre 0.3 et 0.5 près de $\mathrm{Tg}$. Sa valeur absolue est toutefois sensible à la sonde expérimentale et à la méthode d'ajustement, sans omettre la possibilité de faire apparaitre dans le spectre d'autres processus de relaxation. Cette propriété est représentée sur la figure 4 à unẹ température proche de la transition vitreuse pour la m-toluidine mesurée par spectroscopie diélectrique. Il faut toutefois noter que les sondes neutroniques et diélectriques sont de nature différente, (la première mesurant les fonctions d'auto-correlation de densités et la deuxième se rapportant à dynamique orientationnelle des dipôles moléculaires), mais que les temps de relaxation sont très proches et qu'ils conservent la même dépendance en température à l'approche de la transition vitreuse. Les mesures par spectroscopie diélectrique (lorsqu'un dipôle existe) sont plus aisées et moins coûteuses et permettent de dégager dans un premier temps la phénoménologie du problème abordé.

Cet étirement de la relaxation peut être a priori interprété aussi bien en termes d'une distribution de temps de relaxation $\mathrm{g}(\tau)$ dans un milieu hétérogène,

$$
\phi(t)=\int_{0}^{\infty} g(\tau) \cdot e^{-1 / \tau} \cdot d \tau
$$

que résultant de la superposition de relaxations individuelles intrinsèquement étirées dans un milieu homogène. Plusieurs expériences récentes mettant en œuvre des techniques permettant de sélectionner des sous-ensembles de molécules en fonction de la rapidité de leur relaxation (multidimensional NMR, dielectric hole burning, photobleaching) semblent toutefois indiquer que la dynamique est essentiellement hétérogène aux temps longs (cf Sillescu 1999 pour une revue détaillée). 


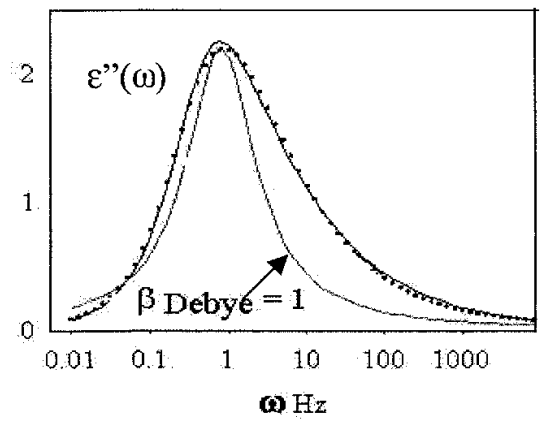

Figure 4: illustration de l'étirement de la fonction de relaxation par rapport à un processus simple de la m-toluidine à une température $(190.6 \mathrm{~K})$ proche de $\mathrm{Tg}$ $(183.5 \mathrm{~K})$. Le trait continu correspond à l'ajustement numérique de la fonction de Cole-Davidson.

\section{3 - lois d'échelle et Superposition Temps-Température.}

Très souvent, on remarque expérimentalement l'existence de lois d'échelle dans la description de la relaxation structurale sous la forme de lois du type $\phi(t, T)=\hat{\phi}(t / \tau(T)$ ) pour les fonctions de relaxation et $\chi \alpha(\omega, T)=\hat{\chi}(\omega \cdot \tau(T)$ pour les spectres de susceptibilité où toute la dépendance en température est contenue dans l'échelle de temps $\tau_{\alpha}$, comme cela est illustré en dessous. La relaxation structurale vérifie ainsi le principe de superposition temps-température bien connu en théorie de la viscoélasticité et appliqué souvent aux propriétés mécaniques des polymères; il s'exprime par une fonction maîtresse du temps, toutefois sur des domaines restreints de temps (ou de fréquence) et de température où la forme des spectres reste semblable (même étirement).

Figure 5: illustration du principe de superposition temps-température sur une fenêtre spectrale donnée pour la enstruction d'une courhe maîtresse

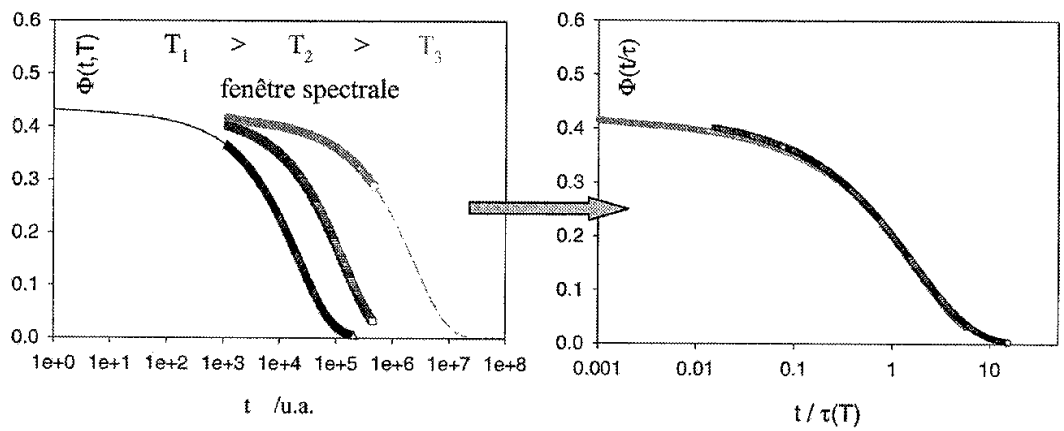

\section{4 - relaxations secondaires $\beta$.}

Sur l'échelle de temps intermédiaire entre les processus microscopiques rapides et la relaxation $\alpha$, on trouve parfois un autre type de relaxation d'amplitude moindre appelée relaxation $\beta$. Elle se manifeste généralement par un excès d'intensité du spectre de diffusion de la lumière ou un pic dans la susceptibilité diélectrique ou encore par RMN. Typiquement, ce type de 
relaxation n'est apparent qu'en dessous du domaine de température marquant la zone de croisement du diagramme d'Angell. Ce pic mis en évidence par Johari et Goldstein(1970), dont il tire aussi son nom $\left(\beta_{\mathrm{JG}}\right)$, serait représentatif d'un mouvement plus local du système. Cependant il n'apparaît pas systématiquement même pour des systèmes très semblables chimiquement, par exemple il est observé dans le cas du toluène mais pas dans celui de la $\alpha$-piccoline, qui sont deux molécules de type van der Waals, rigides et planes. La dépendance en température de la relaxation $\beta_{\mathrm{J}-\mathrm{G}}$ du toluène mesurée par spectroscopie diélectrique est représentée sur la figure 3.

Pour des fréquences supérieures(ou des temps inférieurs), dans la région du minimum des susceptibilités mesurées, on observe un autre processus $\beta_{\text {rapide, }}$ qui n'est pas à proprement parlé une relaxation, mais associé à la relaxation lente, et couramment rattaché à la théorie de couplage de modes (TMC). Cette dynamique rapide se réfère à des mécanismes dans un domaine de fréquence mésocopique de $10-1000 \mathrm{GHz}$, ou quelques $10^{-12} \mathrm{sec}$, c'est-à-dire bien plus rapides que le temps caractéristique de la relaxation $\alpha$ et mais plus lentes que les vibrations d'un atome ou d'une molécule isolée : elles produisent en particulier la contribution quasiélastique des spectres de diffusion neutronique. Dans le domaine du $\mathrm{THz}$, on trouve aussi fréquemment un pic plus ou moins bien résolu, dit pic de Bose (se référer au cours de M.Forêt). Son nom tire son origine de la dépendance en température de son intensité, qui suit la statistique de Bose ; il peut se distinguer dans certain cas (comme sur la figure) d'un pic microscopique apparaissant dans le même domaine de fréquence attribué à un effet de cage, tel qu'il est suggéré dans la TCM. Ce pic boson dans les systèmes vitreux correspond à un excès de densité d'états vibrationnels, que l'on observe aussi dans la chaleur spécifique à très basses températures.

Un point important concernant la compréhension des phénomènes associés à la transition vitreuse est de connaître le rôle joué par l'ensemble de ces processus rapides. Ils peuvent être mesurés par spectroscopie diélectrique, diffusion de la lumière ou de neutrons, à condition de couvrir une gamme en fréquence assez large (Lunkenheimer 2000).

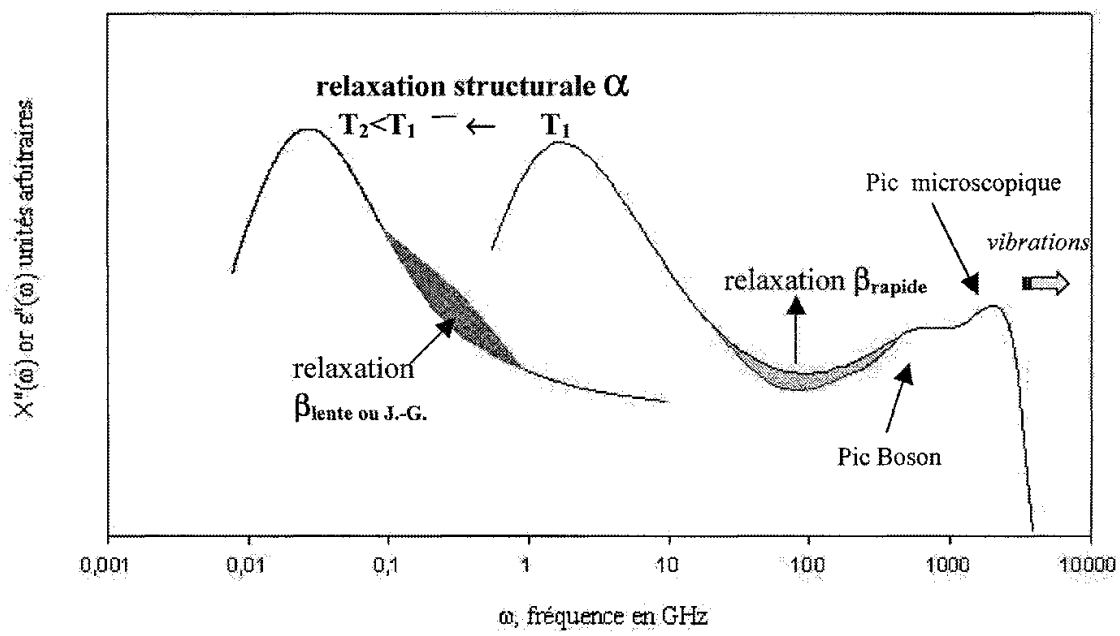

Figure $6:$ représentation schématique des différents processus de relaxation d'un liquide surfondu; exemple de susceptibilité mesurée par diffusion de la lumière, de neutrons ou spectroscopie diélectrique. 


\section{LES LIQUIDES SURFONDUS PAR DIFFUSION DE NEUTRONS.}

La diffusion inélastique de neutrons permet d'étudier simultanément divers mouvements moléculaires ou atomiques ainsi que les directions et les distances sur lesquelles ces mouvements se développent. Pour reprendre l'expression des prix Nobel Brockhouse et Shull en 1994, les neutrons vous disent où les atomes sont et ce qu'ils font.

- Quelle échelle de temps et quelle échelle d'espace ?

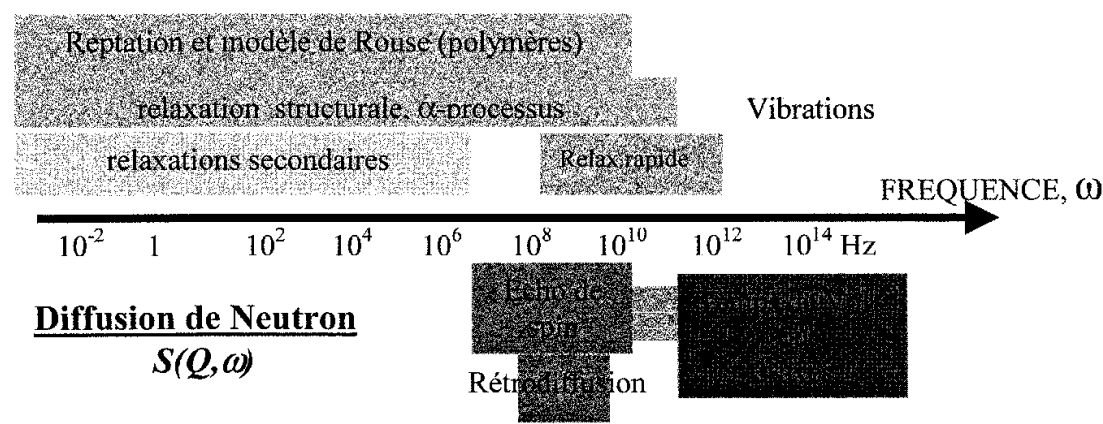

Les liquides surfondus et les verres se prêtent particulièrement bien à ce type d'expérience comme nous l'avons vu précédemment : dans une fenêtre spectrale de quelques picosecondes à la centaine de nanosecondes, et dans un domaine de vecteur d'onde, où les modifications structurales se situent autour du premier pic du facteur de structure $\operatorname{Sm}(\mathrm{Q})$ obtenu par diffraction et illustré sur la figure 7.

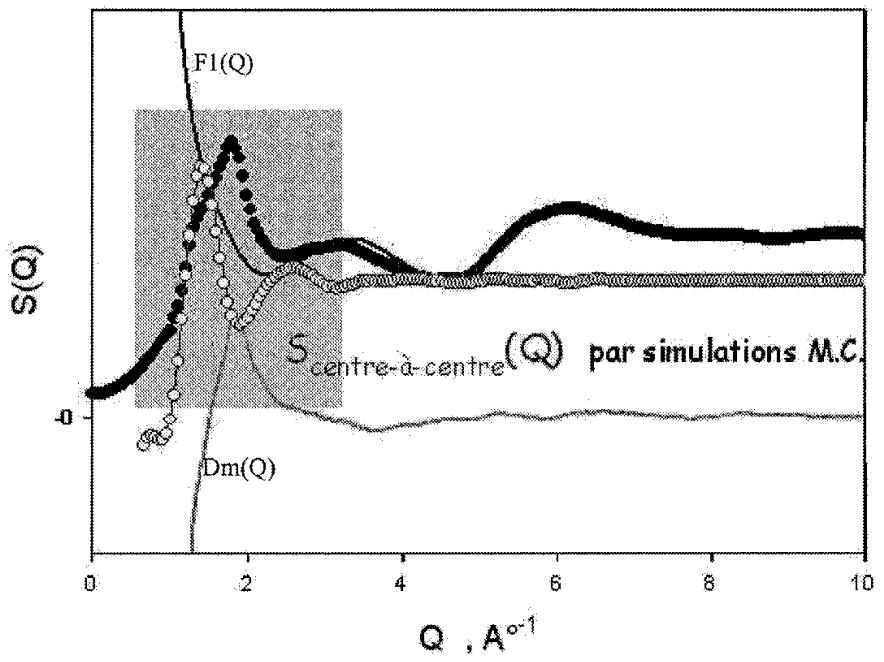

Figure 7: facteur de structure $\mathrm{Sm}(\mathrm{Q})$ du toluène à la Patm et $\mathrm{T}=193 \mathrm{~K}$ composé d'un terme intermoléculaireDm(Q) ( en vett) et d'un terme intramoléculaire fl(Q) (en bleu). Le facteur de structure centre-à-centre (ronds vides) est calculé par simulations numériques Monte-Carlo. (Morineau 1998) 
C'est aussi à cette échelle que l'on soupçonne des réorganisations locales, consécutives au désordre dans les verres et responsables des divers processus de relaxations et à la dynamique vibrationnelle.

- Que mesure-t-on?

La relation entre la structure du liquide et ses propriétés de relaxation se manifeste dans l'évolution temporelle des fonctions d'auto corrélation de densité à différents vecteurs d'onde ; le temps de relaxation moyen par spectre quant à lui présente une forte dépendance avec le vecteur d'onde distincte selon qu'il s'agit de mouvements individuels (ou self) ou collectifs. Ce dernier point se ramène à faire des études d'un système par diffusion respectivement incohérente et cohérente. La quantité mesurée est le facteur de structure dynamique $S(Q, \omega)$,

$$
S(Q, \omega)=\frac{1}{\mathrm{~N}} \int \frac{d \omega}{2 \pi} e^{i \omega t} \sum_{j, k=1}^{N}\left\langle e^{i Q R_{j}(0)} e^{-i Q R_{k}(t)}\right\rangle
$$

qui par une double transformation de Fourier se relie à la fonction de van Hove(1954) $G(r, t)$ :

Facteur de structure

Dynamique $S(Q, \omega)$

$$
\frac{d^{2} \sigma}{d \Omega d E_{f}}=\frac{b^{2} k_{f}}{\hbar k_{i}} S(Q, \omega)
$$

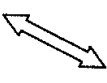

Fonction de corrélation de paire

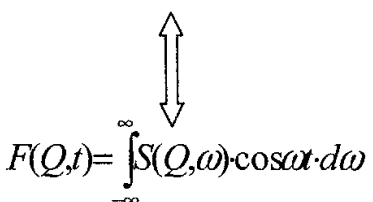
dévendante du temos (Van Hove)

$$
G(r, t)=\frac{1}{2 \pi^{2}} \int_{0}^{\infty} F(Q, t) \cdot \frac{\sin Q r}{Q r} \cdot Q^{2} \cdot d Q
$$

Fonction intermédiaire de diffusion

Ceci permet de associer directement toutes mesures par diffusion de neutrons à des grandeurs théoriques, en particulier à la théorie de couplage de modes, et aux simulations numériques par dynamique moléculaire lorsqu'un potentiel d'interaction réaliste est utilisé, ou par comparaison à un système modèle (Kob 1995). On s'interessera à de la diffusion de paires (collective ou par extension cohérente) lorsque la paire d'atomes considérée est constituée d'atomes différents $(j \neq k) ;$ si $j=k$, on parlera de diffusion individuelle (self ou auto-diffusion ou par extension incohérente). Cependant la mesure expérimentale correspond toujours à la somme de ces deux contributions selon un poids respectif distinct d'une technique de diffusion à l'autre. Ainsi, par temps de vol ou rétrodiffusion :

$$
S^{\exp }(Q, \omega)=\sigma_{\operatorname{coh}} S_{\operatorname{coh}}(Q, \omega)+\sigma_{\text {incoh }} \operatorname{Sincoh}(Q \omega)
$$

par temps de vol avec polarisation de spins (Schärpf 1993) ou echo de spins (Mezei 1972):

$S^{\uparrow \uparrow}(Q, \omega)=\sigma_{\operatorname{coh}} S_{\operatorname{coh}}(Q, \omega)+1 / 3 \sigma_{\text {incoh }} \operatorname{Sincoh}(Q, \omega)$

$S^{\uparrow \downarrow}(Q, \omega)=2 / 3 \sigma_{\text {incoh }} \operatorname{Sincoh}(Q, \omega)$ 


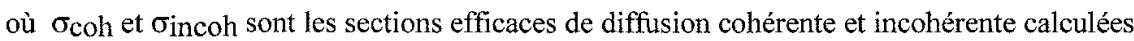
dans la table ci-dessous pour le toluène.

L'association de ces techniques autorise (lorsque les gammes de fréquence ou de temps et de vecteur d'onde le permettent) la connaissance exacte de ces quantités. Cependant, en pratique il sera plus aisé de choisir l'échantillon le mieux adapté à l'étude souhaitée en sélectionnant telle ou telle combinaison d'isotopes (cf table). Sur la figure 8, ce dernier cas est représenté pour le toluène, pour lequel on peut en particulier masquer la participation au spectre de la rotation du groupement méthyl, et selon les cas considérer plus de $80 \%$ de contribution incohérent ou cohérente.

Table: sections efficaces de diffusion cohérente et incohérente du toluène pour différentes compositions isotopiques en barn $\left(1 \mathrm{barn}=100 \mathrm{fm}^{2}\right)$. Les proportions de chaque contribution sont calculées, ce qui permet d'évaluer ce que l'on mesure dans la figure 8.

\begin{tabular}{|c|llll|}
\hline Atome/molécule & $\sigma_{\text {inc }}$ & $\%$ & $\sigma_{\text {coh }}$ & $\%$ \\
& & & & \\
\hline $\mathrm{H}$ & 80.27 & & 1.76 & \\
$\mathrm{D}$ & 2.05 & & 5.59 & \\
$\mathrm{C}$ & 0.00 & & 5.55 & \\
\hline $\mathrm{C}_{6} \mathrm{H}_{5} \mathrm{CD}_{3}$ & 407.50 & 86.3 & 64.42 & 13.7 \\
$\mathrm{C}_{6} \mathrm{D}_{5} \mathrm{CD}_{3}$ & 16.65 & 16.6 & 83.56 & 83.4 \\
\hline $\mathrm{C}_{6} \mathrm{H}_{5} \mathrm{C}$ & 401.35 & 90.0 & 47.65 & 10.0 \\
$\mathrm{C}_{6} \mathrm{D}_{5} \mathrm{C}$ & 10.45 & 13.5 & 66.79 & 86.5 \\
\hline
\end{tabular}
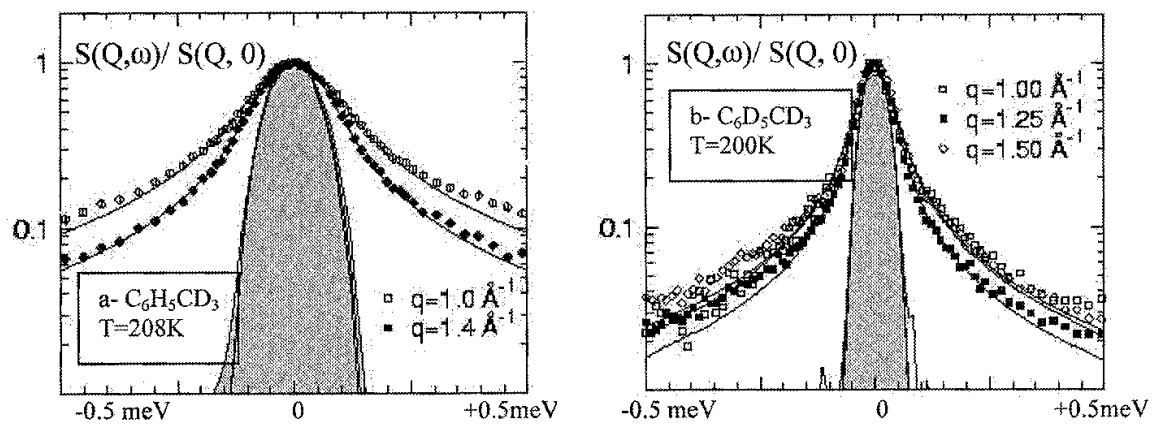

Figure 8: spectres quasiélastiques incohérents (a) et cohérents (b) du Toluène : a- avec un liquide partiellement deutéré afin de supprimer la contribution du groupement méthyl, obtenu par TOF avec $\lambda=$ $5.5 \AA$; b- avec un liquide totalement deutéré, par TOF avec $\lambda=7 \AA$. Les zones assombries correspondent à la résolution instrumentale selon ces deux longueurs d'onde. 
Les spectromètres utilisables pour la diffusion quasiélastique et inélastique de liquides surfondus et des verres sont de plusieurs types :

-les temps de vol, (Time-Of-Flight) mesurent $S(2 \theta, \omega)$, selon l'angle de diffusion $2 \theta$ qu'il faut ensuite convertir en $S(Q, \omega)$, pour des phénomènes dans la gamme de la picoseconde. Plusieurs instruments de ce type sont accessibles dans les centres d'étude (ILL, LLB, HMI, Jülich, ISIS....) avec des configurations variables (longueurs d'onde, forme de la résolution instrumentale, gamme de gain d'énergie, détecteurs..). à la fois.

-les trois-axes (Triple-axis), qui donnent accès explicitement à $\mathrm{S}(\mathrm{Q}, \omega)$ mais pour un $\mathrm{Q}$

-la rétrodiffusion (BackScattering), avec des résolutions de l'ordre de la nanoseconde, fournissent aussi $\mathrm{S}(\mathrm{Q}, \omega)$ mais dans un domaine d'énergie plus restreint $(-15$ à $15 \mu \mathrm{eV})$; l'énergie des neutrons incidents étant beaucoup plus grande que le gain ou la perte d'énergie, le transfert de moment à un angle donné est maintenu constant.

-l'écho de spins (Neutron Spin-Echo), qui utilisent les propriétés magnétiques du neutron pour une mesure de la fonction intermédiaire $\mathrm{F}(\mathrm{Q}, \mathrm{t})$ directement résolue en temps. Le domaine accessible s'étend de quelsues picosecondes à la centaine de nanoseconde, selon la gamme de vecteur d'onde désirée.

-le temps de vol utilisant la polarisation de neutrons, qui sépare les contributions cohérente et incohérente, beaucoup plus rare et avec une résolution en énergie bien moins bonne (de l'ordre de $0.2 \mathrm{meV}$ ).

Il faut tenir de ces diverses caractéristiques si l'on veut étendre le plus possible le domaine en temps des processus de relaxation qui se développent dans les liquides visqueux. La question se pose alors du raccordement entre instruments différents : il est nécessaire de réaliser des transformations de Fourier comme suit :

$$
S(Q, \omega)=\frac{1}{2 \pi} \int_{-\infty}^{+\infty} \exp (-i \omega t) F(Q, t) d t
$$

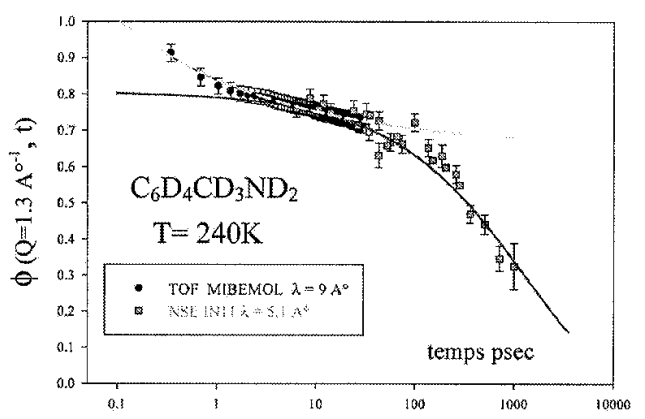

Figure $9:$ raccordement de mesures entre - un spectromètre à temps de vol (ici mibemol au LLB) aux temps courts , après une transformation de Fourier, et -une mesure des fluctuations de densités par écho de spins (ici sur INII à l'ILL) dans le cas de la m-toluidine à $240 \mathrm{~K}$. Ceci permet de réaliser divers ajustements numériques de fonctions, aux temps courts par les équations de couplage de modes et aux temps plus longs par la loi de Kohlrausch. 
cependant il ne faut pas oublier que les mesures ne correspondent pas tout à fait aux mêmes observables en particulier entre temps de vol et echo de spin (cf figure 9). Il est alors utile de reccourir à des raisons physiques ou des formules théoriques pour justifier tel ou tel recalage. (Tölle 2002)

- Difficultés de l'analyse des données expérimentales.

Pour obtenir ce facteur de structure dynamique, si clairement défini, il faut effectuer tout d'abord un certain nombre de corrections aux données expérimentales brutes et s'assurer du maintien du même nombre de centres diffusants et de la même géométrie d'un spectre à l'autre. En premier lieu et pour chaque série d'expériences, il faut -tenir compte de l'efficacité des détecteurs, -normaliser les intensités au flux incident, et à un échantillon de Vanadium (ou de quartz dans le cas de neutrons polarisés) dans les mêmes conditions que le système étudié, soustraire les contributions de la cellule vide ou du cryostat (ce qui revient à négliger délibérément tous termes croisés), et -calculer les corrections dûes à l'atténuation du flux et à l'absorption des couches de matière traversées. Exceptés quelques montages particuliers (tel que les cellules à très haute pression,etc...), l'ensemble de ces corrections sont prises en compte de façon standard dans les programmes proposés dans les différents centres neutroniques.

Une complication supplémentaire provient de phénomènes de diffusion multiple incontrôlable et inévitable, liée à la convolution du spectre par lui-même ; son intensité varie avec le vecteur d'onde (et donc la longueur d'onde sélectionnée), et est d'autant plus forte que $Q$ est petit. Cette correction n'a rien de standard ou de systématique, néanmoins plusieurs modes opératoires sont proposés : -effectuer des simulations Monte-Carlo à partir d'un spectre modèle jusqu'à la reconstitution du spectre expérimental par itérations successives (Wüttke 2002), réaliser une série d'expériences équivalentes avec des longueurs d'onde différentes (Russina 2000), -se mettre dans les meilleures conditions pour minimiser cet effet en optimisant la transmission de l'échantillon (>90\%).

Enfin, pour traiter un spectre de diffusion, il faut tenir compte du rapport signal/bruit et de la forme de la résolution du spectromètre $R(\omega)$.

$$
S \exp (Q, \omega)=\text { Sthéorique }(Q, \omega) \otimes R(\omega)
$$

si on a un modèle théorique ou empirique, dont on ajustera les paramètres lors de la convolution à la résolution, ou bien numériquement, après transformation de Fourier

$$
\text { T.F. }[\operatorname{Sexp}(\mathrm{Q}, \mathrm{w})]=\mathrm{S} \text { théorique }(\mathrm{Q}, \mathrm{t}) \cdot \mathrm{R}(\mathrm{t})
$$

indépendamment d'un modèle, et on divisera alors par la résolution exprimée dans le temps Ce dernier mode opératoire est particulièrement valable quand le spectre expérimental est proche de la résolution. Il reste encore une dernière difficulté dans le raccordement entre instrument résultant du choix de la coupure en fréquence et du domaine de TF.

\subsection{Relaxation en deux étapes : processus $\beta$ rapide et relaxation $\alpha$}

En accord avec les predictions de la théorie de couplage de modes, antérieures aux premières expériences, la décroissance de la fonction de relaxation s'effectue en deux étapes dans le domaine de temps (ou de fréquences) de la diffusion de neutrons, comme cela est illustré sur la figure 9 et shématiquement sur la figure 10. Lorsque la température diminue, on distingue, après un domaine transitoire correspondant aux vibrations du système, un premier régime à temps

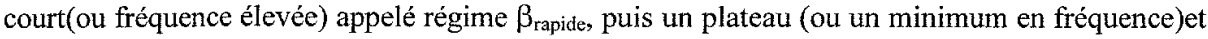


enfin une décroissance jusqu'à zéro (ou un pic) de la relaxation structurale. Sur chacun de ces trois régimes selon leur évolution avec la température (ou la densité), on pourra appliquer des lois de la théorie, qui seront développées plus bas dans le dernier chapitre.

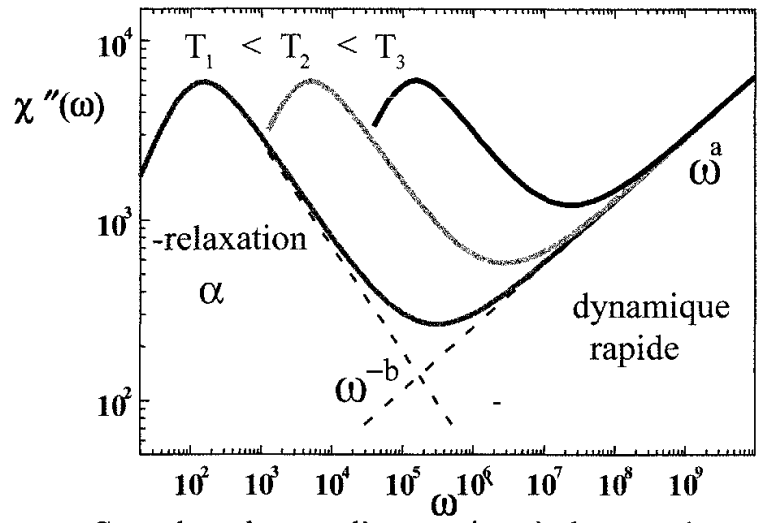

Figure $10:$ scenario qualitatif de la théorie de couplage de modes; sur la base d'une équation maîtresse autour du minimum : $\chi(\omega)=\chi_{\min }\left\{b^{*}\left(\omega \omega_{\min }\right)^{\mathrm{a}}+\mathrm{a}^{*}\left(\omega_{\min } / \omega\right)^{-b}\right\} /(\mathrm{a}+\mathrm{b})$

Cependant, lorsque l'on se situe à des températures où la relaxation structurale est nettement sortie de la fenêtre expérimentale, c'est-à-dire au-delà de quelques nanosecondes (dans la zone de cross over achurée de la figure 2) jusqu'à $\mathrm{Tg}$ et en-dessous, il n'existe aucune prédiction de comportement. On doit alors s'intéresser à l'évolution temporelle du facteur de Debye -Waller (i.e. la portion élastique des spectres) ou bien mesurer un déplacement carré moyen restreint aux temps des résolutions expérimentales (soit au maximum à la nsec).

\subsection{Principe de superposition temps-température.}

La détermination complète d'une fonction de relaxation sur un large intervalle de temps ou de fréquence, bien que possible conceptuellement, rencontre de nombreuses difficultés expérimentales; en effet le processus est souvent explorée dans un domaine de temps ou de fréquence restreint, ce qui conduit à associer plusieurs mesures à des températures différentes pour reconstituer la fonction complète sous la forme d'une courbe réduite ou maîtresse par rapport à une température de référence.

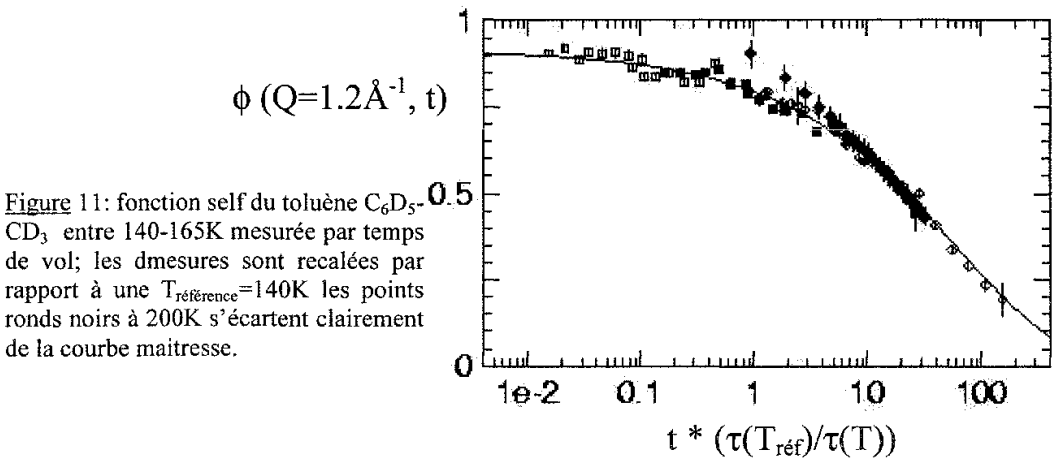


En première approximation et sur une domaine de température limitée, les données expérimentales suivent le principe de superposition temps-température: les mesures à différentes températures des fluctuations de densité à un vecteur d'onde donné se superposent lorsque l'axe du temps est réajusté par $\tau\left(\mathrm{T}_{\text {ref }}\right) / \tau(\mathrm{T})$ (cf figure 11). Cette opération se vérifie tant sur les fluctuations de densités individuelles que de paires dans la fenêtre spectrale de la diffusion de neutrons, et ce pour de nombreux systèmes liquides, fondus de polymères, liquides ioniques ou métalliques. Cette propriété permet d'outrepasser la limitation des analyses due à un domaine de temps expérimental restreint ; il est possible de réaliser un ajustement numérique de la fonction de Kohlrausch $\phi_{\mathrm{Q}}(\mathrm{t})=\mathrm{A}_{\mathrm{Q}} \exp \left(-(t / \tau)^{\beta \mathrm{Q}}\right)$ pour chaque vecteur d'onde afin de déterminer un exposant $\beta_{Q}$ et un facteur préexponentiel $A_{Q}$, sur l'hypothèse (à verifier ensuite par itération successive) que ces deux paramètres sont indépendants de la température dans la plage étudiée ; c'est alors la première étape pour déterminer avec moins d'ambiguité le temps de relaxation $\tau(\mathrm{Q}, \mathrm{T})$ dont on se servira par la suite.

\subsection{Dépendance en $Q$ de l'auto-diffusion et de la diffusion de paire.}

Si l'on s'interesse mainten nt. à la dependance des spectres avec les vecteurs d'onde, on remarque tout de suite des comportements différents selon que l'on s'interesse à la diffusion cohérente ou incohérente du sysème (figure $8 \mathrm{a}$ et b). On constate en effet que les spectres incohérents sont plus larges que les spectres cohérents et que la largeur des premiers diminue beaucoup plus vite avec Q. (Prenons l'exemple simple de particules échangeant leur positions, on voit bien que tous les déplacements ne contribueront pas à la décorrélation des fonctions de paire). Les figures 12 et 13 représentent respectivement les temps de relaxation $\tau(\mathrm{Q}, \mathrm{T})$ incohérents et cohérents, définis soit en utilisant la principe de STT soit en raccordant divers domaine spectraux de différents instruments.

Sur la figure 12, on constate une dépendance en $\mathrm{Q}^{-\mathrm{n}}$, avec $\mathrm{n}$ de l'ordre de 2 ; les mesures sur un large domaine de $\mathrm{Q}$ obtenues par temps de vol font clairement apparaître une fonction étirée pour laquelle l'exposant d'étirement ( Kohlraush) est constant et de l'ordre de .65; ici la variation semble continue, alors que dans le cas des polymères, deux régimes sont observés, en $Q^{-2 / \beta} \mathrm{K}$ à petits $Q$ et $Q^{-2}$ à grands vecteurs d'onde (Arbe et al 1998-2002).

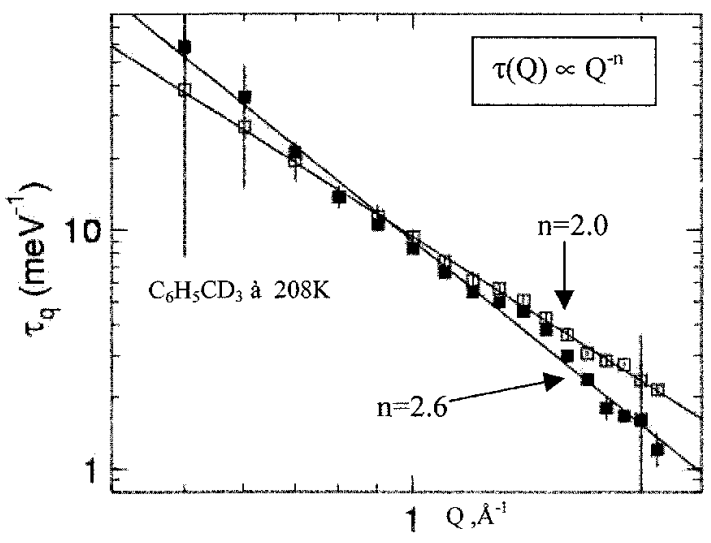

Figure $12:$ dépéndance en vecteur d'onde du temps de relaxation self $d u$ toluène à $208 \mathrm{~K}$, calculé à partir de la des spectres de la figure 8 
Pour la diffusion cohérente de systèmes atomiques simples, de Gennes (1959) avait conclut que le temps de relaxation de caractère translationnel à divers vecteurs d'onde est proportionnel au facteur de structure $S(Q)$ divisé par $Q^{2}$; le cas de l'argon liquide (Sköld 1972) illustre assez bien ce qui est appelé le rétrécissement de de Gennes (de Gennes narrowing). Pour les liquides moléculaires, tel que le toluène, on constate que la relaxation structurale mesurée par échos de spins présente une dépendance avec le vecteur d'onde Q qui n'est pas proportionnelle au facteur de structure moléculaire $\operatorname{Sm}(\mathrm{Q}) / \mathrm{Q}^{2}$, mais suit le facteur de structure associé au centre de masse des molécules $\mathrm{S}_{\mathrm{C}-\mathrm{C}}(\mathrm{Q}) / \mathrm{Q}^{2}$ (se référer à la figure 13 ). Ainsi le maximum de ce temps de relaxation se situe au maximum de $\mathrm{S}_{\mathrm{C}-\mathrm{C}}(\mathrm{Q}) / \mathrm{Q}^{2}$ et non à celui du facteur de structure moléculaire $\operatorname{Sm}(Q) / Q^{2}$. Quant à l'exposant $\beta_{\mathrm{K}}$ de la fonction étirée, il présente un faible oscillation autour de ce maximum avec une valeur moyenne de l'ordre de .54 .

La dynamique de ces molécules aromatiques rigides est donc dominée par les mouvements translationnels, sans influence significative des contributions réorientationnelles des plans, ni de la présence de liaisons hydrogène. Il faut noter que le facteur de structure lié au centre de masse n'est pas observé directement expérimentalement $(\mathrm{Sm}(\mathrm{Q})$ résulte de la combinaison des différents facteurs de structures partiels interatomiques), mais il peut être obtenu par simulation numérique. Ce rétrécissement de de Gennes à l'échelle moléculaire dans un liquide surfondu est d'autant plus remarquable que dans ce régime la présence d'effets mémoire conduit à la décroissance des fluctuations de densités selon des lois en exponentielle étirée et que les observables dynamiques dépendent à la fois de degrés de liberté translationnel et rotationnel. De nouveaux résultats semblent mettre en évidence plus clairement ces derniers à des vecteurs d'onde plus élevés $\left(2.4 \mathrm{~A}^{\circ-1}\right)$ et leur couplage peut être plus aisément caractérisé par simulations numériques de la Dynamique Moléculaire.

Ce comportement a égalemet été observé pour des liquides ioniques (figure 13) par Mezei et al (1991) et pour des polymères (Arbe 1998)

Malheureusement l'échelle de temps expérimental limitée à quelques nanosecondes de la diffusion neutronique ne permet pas de sonder cette relation dans le cas où la relaxation structurale deviendrait très lente, c'est-à-dire à l'approche de la transition vitreuse.

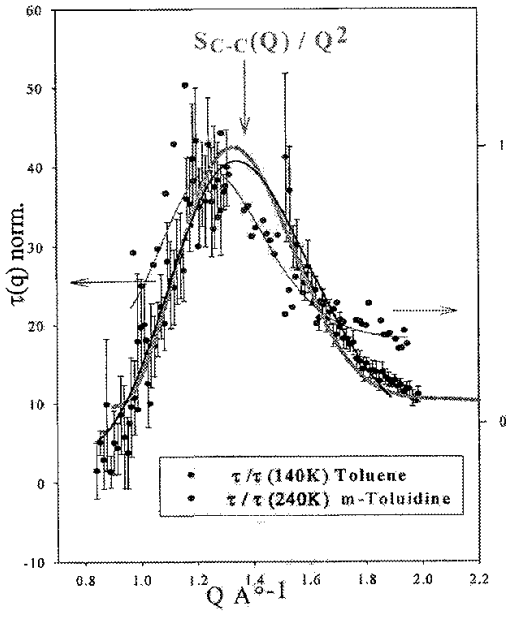

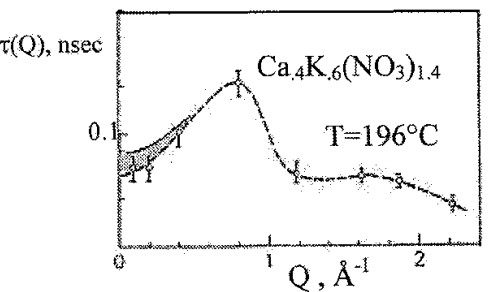

Figure 13: illustration du rétrécissement de de Gennes pour des liquides moléculaires aromatiques tels que le toluène et la mtoluidine, et pour un liquide constitué de différentes espèces ioniques (Mezei 1991). L' interprétation reste toutefois distincte : dans le premier cas, la relation au $\mathrm{S}(\mathrm{Q})$ met en évidence le mouvement translationnel du système, dans le deuxième, l'espèce chimique la plus lente. 


\section{THEORIE DE COUPLAGE DE MODES ET LIQUIDES SURFONDUS.}

\subsection{Présentation de la théorie}

Puisque la transition vitreuse est clairement identifiée comme un phénomène cinétique qui trouve son origine dans le ralentissement considérable de la dynamique d'un liquide surfondu lorsque celui-ci est refroidi ou comprimé, on peut raisonnablement espérer la comprendre dans le cadre des approches théoriques qui ont été progressivement élaborées depuis le début des années mil neuf cent soixante pour l'étude de la dynamique lente des phases condensées caractérisées par des degrés de liberté fortement corrélés. C'est sur la base de cette intuition qu'a été particulièrement développée l'application à l'état liquide surfondu d'une de ces approches, celle dite de couplage de modes.

D'une façon générale, on appelle théorie de couplage de modes une théorie dans laquelle on cherche à rendre compte de la dynamique d'un système physique uniquement à partir de variables lentes bien choisies et de leurs produits (il suffit généralement de se limiter aux termes quadratiques). Il existe plusieurs façons de développer un formalisme à partir de cette hypothèse initiale, mais, en dernier ressort, une théorie de couplage de modes permet d'obtenir un système d'équations auto-cohérent pour les fonctions de corrélation des variables lentes, dont l'évaluation ne nécessite que la connaissance d'un petit nombre de grandeurs statiques. C'est cette structure de la théorie qui lui permet de rendre compte de propriétés dynamiques non triviales. Avant de poursuivre, notons qu'en général on ne sait pas estimer a priori la qualité d'une approche de couplage de modes et que les hypothèses et approximations effectuées pour l'obtenir sont en grande partie incontrôlées. Ce n'est guère que par son succès à rendre compte d'un phénomène physique qu'une telle approche est validée. L'exemple le plus simple, mais déjà très riche du point de vue de la physique, de mise en œuvre des approches de couplage de modes pour l'étude de la dynamique des liquides surfondus et de la transition vitreuse est l'application aux liquides atomiques du formalisme dit de traitement auto-cohérent de la relaxation des courants (self consistent current relaxation theory). On considère alors un ensemble de $N$ particules classiques identiques de masse $m$, contenues dans un volume $V$ (on note $n=N / V$ leur densité numérique) et interagissant suivant un potentiel de paire à symétrie sphérique. Définissant la fluctuation de densité numérique de vecteur d'onde $\mathbf{q}$,

$$
\rho_{\mathrm{q}}=\sum_{k=1}^{N} e^{i \mathrm{q} \mathbf{r}_{k}(t)},
$$

où $\mathbf{r}_{k}$ est la position de la particule $k$, et la fonction d'autocorrélation normalisée associée,

$$
\phi_{q}(t)=\frac{\left\langle\rho_{q}(t) \rho_{-q}\right\rangle}{\left\langle\rho_{q} \rho_{-q}\right\rangle}=\frac{\left\langle\rho_{q}(t) \rho_{-q}\right\rangle}{N S_{q}},
$$

où $S_{q}$ est le facteur de structure du fluide, on arrive, dans le cadre de cette approche, aux équations suivantes :

$$
\ddot{\phi}_{q}(t)+v_{q} \dot{\phi}_{q}(t)+\Omega_{q}{ }^{2} \phi_{q}(t)+\Omega_{q}{ }^{2} \int_{j} d \tau m_{q}(t-\tau) \dot{\phi}_{q}(\tau)=0,
$$

avec

$$
m_{q}(t)=\frac{n S_{q}}{16 \pi^{3} q^{2}} \int d^{3} \mathbf{k} S_{k} S_{|\mathbf{q}-\mathbf{k}|}\left(\left(\mathbf{k} c_{k}+(\mathbf{q}-\mathbf{k}) c_{|\mathbf{q}-\mathbf{k}|}\right) \frac{\mathbf{q}}{q}\right)^{2} \phi_{k}(t) \phi_{|\mathbf{q}-\mathbf{k}|}(t),
$$




$$
\Omega_{q}^{2}=\frac{q^{2} k_{B} T}{m S_{q}}
$$

et les conditions initiales $\phi_{q}(0)=1$ et $\dot{\phi}_{q}(0)=0 . T$ est la température et $k_{B}$ désigne la constante de Boltzmann. $v_{q}$ est un coefficient de friction modélisant de façon simplifiée la dissipation associée aux processus vibrationnels rapides et $c_{q}$ est la fonction de corrélation directe qui vérifie $n c_{q}=1-1 / S_{q}$.

Ces équations forment la base d'une théorie de couplage de modes de la transition vitreuse pour des raisons qui apparaitront à la section suivante. Elles constituent la mise en équation d'un mécanisme de rétroaction proposé en premier par Geszti (1983) comme source possible de ralentissement drastique de la dynamique des liquides. Elles prennent également en compte deux phénomènes considérés comme importants dans la description phénoménologique de la dynamique des liquides denses. Le premier est l'effet de cage qui correspond au fait qu'une particule dans un fluide dense est confinée durablement dans une cage formée de ses premières voisines et qu'elle oscille dans celle-ci au gré de multiples collisions avant de parvenir à s'en échapper. Le second est le phénomène de contre-courant (back flow) qui traduit le fait que, dans un milieu dense, compte tenu du faible volume spécifique disponible pour chaque particule, le mouvement d'une particule hors de sa cage doit nécessairement s'accompagner d'une mouvement d'ensemble de celles qui l'entourent pour d'une part lui laisser libre le passage et d'autre part occuper la région laissée vacante par son départ.

Pour finir, signalons que des équations analogues à (3) ont pu être dérivées qui visent à décrire l'évolution d'autres grandeurs importantes de la dynamique d'un liquide simple, comme les fluctuations de densité d'une particule marquée, ou encore la dynamique de systèmes plus complexes comme les mélanges binaires de liquides atomiques ou des systèmes moléculaires rigides ou flexibles.

\subsection{Prédictions de la théorie de couplages de modes}

\subsubsection{Propriétés génériques d'une famille d'équations}

Les équations de couplage de modes (3) peuvent être généralisées pour définir une famille d'équations vérifiant :

$$
\begin{gathered}
\ddot{\phi}_{i}(t)+\Gamma_{i} \Omega_{i} \dot{\phi}_{i}(t)+\Omega_{i}{ }^{2} \phi_{i}(t)+\Omega_{i}{ }^{2} \int d \tau m_{i}(\mathbf{V} ; \varphi(t-\tau)) \dot{\phi}_{i}(\tau)=0, \\
m_{i}(\mathbf{V} ; \mathbf{f})=\sum_{j=1}^{\infty} \sum_{k_{1}, \ldots, k_{j}} V_{k_{1}, \ldots, k_{j}} f_{k_{i}} \cdots f_{k_{j}}, \\
\phi_{i}(0)=1,
\end{gathered}
$$

que l'on étudie lorsque varie le vecteur $\mathbf{V}=\left(V_{k_{1}, \ldots, k_{j}}\right)$, dont les composantes, supposées positives ou nulles, sont appelées vertex.

Cette famille d'équations présente un certain nombre de propriétés génériques non triviales.

- Bifurcation entre deux régimes asymptotiques 
La limite à temps infini des solutions de l'équation (4) est la plus grande des solutions dans $[0,1[$ de l'équation :

$$
\frac{f_{i}}{1-f_{i}}=m_{i}(\mathbf{V} ; \mathbf{f}) \text {. }
$$

Sur un chemin joignant un voisinage de l'origine à un voisinage de l'infini dans l'espace des vertex, il y a une transition entre un domaine où l'unique solution de (5) est la solution triviale nulle et un domaine où il existe une solution non nulle.

La frontière entre ces deux domaines est appelée hypersurface critique et correspond à des valeurs $\mathbf{V}^{c}$ des vertex et $\mathbf{f}^{c}$ des asymptotes telles que soient vérifiées simultanément l'équation (5) et

$$
\operatorname{dét}\left[\frac{1}{\left(1-f_{i}^{c}\right)^{2}} \delta_{i j}-\frac{\partial m_{i}}{\partial f_{i}}\left(\mathbf{V}^{c} ; \mathbf{f}^{c}\right)\right]=0 .
$$

Il existe essentiellement deux types de transitions. Si le vecteur $\mathbf{f}^{c}$ est nul à la transition, la transition est dite de type $A$. En revanche, si le vecteur $\mathbf{f}^{c}$ prend de manière discontinue une valeur non nulle, la transition est dite de type $\mathrm{B}$. Dans la suite, on se limitera à ces dernières qui sont les seules pertinentes pour la physique des liquides surfondus.

- Singularité au voisinage de la transition

Au voisinage d'un point générique de l'hypersurface critique, on peut montrer qu'il existe un paramètre $\sigma$, appelé paramètre de séparation, qui quantifie la distance à l'hypersurface critique (il est nul sur cette hypersurface, négatif dans le domaine des couplages faibles et positif dans le domaine des couplages forts) et tel que $f_{i}$ vérifie

$$
f_{i}= \begin{cases}0 & \text { si } \sigma<0, \\ f_{i}^{c}+h_{i} \sqrt{\frac{\sigma}{1-\lambda}} & \text { si } \sigma \geq 0 .\end{cases}
$$

Le paramètre $\lambda$ (dont on admet généralement qu'il peut prendre toute valeur comprise entre $1 / 2$ et 1 pour une transition de type B) est appelé paramètre d'exposant. C'est le paramètre le plus important dans la mesure où il détermine à lui seul nombre de propriétés non triviales des solutions des équations (4).

$\sigma$ est une fonction linéaire des vertex, il y a donc une singularité en racine carrée à la traversée de l'hypersurface critique, et on appelle les coefficients $h_{i}$ associés les amplitudes critiques.

- Théorème de réduction

Au voisinage de l'hypersurface critique, dans le domaine de temps où $\phi_{i}$ prend des valeurs proches de $f_{i}^{c}$, on a à l'ordre dominant

avec $G(t)$ donné par

$$
\phi_{i}(t)=f_{i}^{c}+h_{i} G(t)
$$

$$
\sigma+\lambda G(t)^{2}-\frac{d}{d t} \int_{0} d \tau G(t-\tau) G(\tau)=0
$$


D'où le nom de théorème de réduction donné à cette propriété : au voisinage de leur valeur asymptotique critique, les fonctions $\phi_{i}$ présentent, à un facteur multiplicatif près, une allure en fonction du temps indépendante de l'indice $i$.

- Allure des solutions et échelles de temps

On peut déterminer à partir des résultats précédents l'allure des fonctions $\phi_{i}(t)$ et des susceptibilités $\chi_{i}^{\prime \prime}(\omega)$ associées. Pour cela, il suffit de résoudre de façon approchée l'équation d'échelle (8). On ne s'intéresse ici qu'au cas où $\sigma$ est négatif.

On trouve alors une première échelle de temps importante

$$
t_{\sigma} \approx t_{0}|\sigma|^{-1 / 2 a} \text {, }
$$

où $t_{0}$ est une constante déterminée par le raccordement de ce régime avec le domaine des temps microscopiques. Cette échelle de temps, qui diverge à l'approche de la bifurcation, correspond au régime dit de décroissance critique, qui décrit la façon dont $\phi_{i}$ approche $f_{i}^{c}$ par les valeurs supérieures. $a$ doit être la solution positive et inférieure à 0.395 de l'équation $\lambda=G(a)$, où

$$
G(x)=\frac{\Gamma(1-x)^{2}}{\Gamma(1-2 x)}
$$

( $\Gamma$ représentant la fonction Gamma d'Euler). Dans ce régime, on obtient les comportements suivants :

$\phi_{i}\left(t<<t_{\sigma}\right) \approx f_{i}^{c}+h_{i} \sqrt{\sigma}\left(\frac{t_{\sigma}}{t}\right)^{a} ; \chi_{i}^{\prime \prime}\left(\omega \gg t_{\sigma}^{-1}\right) \approx h_{i} \sin \left(\frac{\pi}{2} a\right) \Gamma(1-a) \sqrt{\sigma}\left(\omega t_{\sigma}\right)^{a}$.

Un second domaine est déterminé par l'échelle de temps

$$
\tau_{\sigma} \approx t_{0} B^{-1 / b}|\sigma|^{-1 / 2 a-1 / 2 b},
$$

avec $b$ positif, inférieur à 1 et solution de $\lambda=G(-b)$, et $B$ qui est une constante déterminée également par la valeur de $\lambda$. Cette échelle de temps diverge à l'approche de la bifurcation plus rapidement que $t_{\sigma}$ et on peut montrer qu'elle caractérise la décroissance de $\phi_{i}$ de $f_{i}^{c}$ à 0 . Dans ce régime, on a

$$
\phi_{i}(t) \approx \bar{\phi}_{i}\left(t / \tau_{\sigma}\right), \chi_{i}^{\prime \prime}(\omega) \approx \bar{\chi}_{i}^{\prime \prime}\left(\omega \tau_{\sigma}\right)
$$

et lorsque $\phi_{i}$ a une valeur proche de $f_{i}^{c}$, on trouve la loi de von Schweidler

$$
\phi_{i}(t) \approx f_{i}^{c}-h_{i}\left(\frac{t}{\tau_{\sigma}}\right)^{b} ; \chi_{i}^{\prime \prime}(\omega) \approx h_{i} \sin \left(\frac{\pi}{2} b\right) \Gamma(1+b)\left(\omega \tau_{\sigma}\right)^{-b} .
$$

L'allure des solutions des équations (4) se déduit aisément de ces résultats : au voisinage de l'hypersurface critique, elles se caractérisent par une décroissance en deux temps séparés par un domaine où elles restent quasiment à la valeur de l'asymptote critique, ce plateau étant d'autant plus long que les vertex sont proches de leur valeur critique. La première étape de la décroissance est essentiellement invariable, tandis que la décroissance finale de $\phi_{i}$ se fait sur l'échelle de temps $\tau_{\sigma}$. Au voisinage du plateau, correspondant à l'échelle de temps $t_{\sigma}$, l'allure de toutes les fonctions est donnée par une unique fonction d'échelle avec une amplitude proportionnelle à $\sqrt{\sigma}$. 
Quant aux susceptibilités, elles font apparaître deux pics. Le pic de plus haute fréquence ne varie quasiment pas. Le pic de plus basse fréquence correspond à la deuxième étape de la décroissance de $\phi_{i}$ et est donc caractérisé par une fréquence de l'ordre de $\omega_{\sigma}=\tau_{\sigma}^{-1}$. Entre les deux pics un minimum apparaît à une fréquence de l'ordre de $\omega_{\sigma}{ }^{\prime}=t_{\sigma}{ }^{-1}$, dont la forme est donnée ici aussi par une unique fonction d'échelle avec une amplitude proportionnelle à $\sqrt{\sigma}$.

\subsubsection{Application à la théorie auto-cohérente de relaxation des courants}

Les équations (3) décrivant l'évolution des fluctuations de densité obtenues dans le cadre de la théorie auto-cohérente de relaxation des courants appartiennent à la famille d'équations considérée à la section précédente, les vertex étant dans ce cas déterminés uniquement par le facteur de structure statique et la densité du liquide étudié. Nous allons voir que les propriétés exposées précédemment permettent d'envisager cette théorie de couplage de modes comme une approche possible du phénomène de transition vitreuse et conduisent à un certain nombre de prédictions non triviales susceptibles d'être vérifiées expérimentalement.

Lorsqu'un liquide simple est refroidi ou comprimé le long d'un chemin thermodynamique donné, sa structure et donc les vertex changent continûment de telle sorte que globalement l'intensité du couplage va croissant (l'effet dominant étant l'augmentation de l'aire du pic principal du facteur de structure statique). Une bifurcation du type de celle décrite plus haut (nécessairement de type B car la fonctionnelle de couplage de modes est quadratique) peut alors éventuellement se produire en un point de ce chemin. Cette transition purement dynamique est appelée transition vitreuse idéale et sépare le domaine où $\lim _{t \rightarrow \infty} \phi_{q}(t)=0$ qui correspond à l'état liquide de celui où $\lim _{t \rightarrow \infty} \phi_{q}(t)=f_{q} \neq 0$ qui correspond à l'état de verre idéal. Cette terminologie repose sur le fait que, lorsque $\phi_{q}$ ne décroît pas à zéro, le spectre des fluctuations de densité présente une contribution élastique

$$
\phi_{q}{ }^{\prime \prime}(\omega)=\pi f_{q} \delta(\omega)+\text { fonction continue de } \omega
$$

caractéristique d'un solide dont $f_{q}$ est le facteur de Debye-Waller. Celui-ci étant une fonction continue de $q$, il s'agit d'un solide amorphe que l'on convient d'appeler un verre idéal. Le terme idéal vient ici signaler le fait que la transition est déterminée de façon univoque par les propriétés d'équilibre du système et donc ne dépend pas des conditions de refroidissement ou de compression, à la différence de la transition vitreuse expérimentale. L'existence de fluctuations figées est une signature typique du phénomène de brisure d'ergodicité. $f_{q}$ est donc également appelé paramètre de non-ergodicité ou encore paramètre d'Edwards-Anderson du nom des auteurs qui ont introduit une grandeur similaire comme paramètre d'ordre dans une théorie de verres de spins.

En plus d'apparaître discontinûment à la transition, le paramètre de non-ergodicité présente un comportement critique à l'entrée dans l'état vitreux idéal. En effet, si la variation d'une grandeur intensive $X$ caractérise une trajectoire donnée dans le diagramme de phase du liquide étudié (typiquement, les trajectoires considérées expérimentalement sont des isobares à la pression atmosphérique paramétrées par la température : on peut alors choisir $X \equiv T$ ), alors les grandeurs statiques étant des fonctions régulières de $X$, il en va de même des vertex. On peut donc linéariser le paramètre de séparation $\sigma$ au voisinage du point $X=X_{c}$ : 


$$
\sigma \approx C_{X} \frac{X-X_{c}}{X_{x}},
$$

et, en reportant dans (7), on obtient alors dans l'état vitreux idéal le comportement singulier

$$
f_{q}-f_{q}^{c} \propto \sqrt{\left|X-X_{c}\right|} .
$$

Les prédictions pour la dynamique découlent directement des résultats de la section précédente. On trouve donc, dans la phase liquide ergodique, une relaxation en deux étapes dont un paramètre essentiel est le paramètre d'exposant $\lambda$, qui seul détermine nombre de propriétés asymptotiques des solutions des équations de la théorie, en particulier les exposants $a$ et $b$.

C'est au sujet de la dynamique au voisinage du plateau, communément appelée processus $\beta$ dans la littérature, que la théorie fait les prédictions les plus importantes. C'est le cas de la propriété de factorisation qui découle directement du théorème de réduction

$$
\phi_{q}(t)-f_{q}^{c} \propto G(t), \chi_{q}^{\prime \prime}(\omega) \propto \omega G^{\prime \prime}(\omega),
$$

où la fonction $G$, indépendante de $q$, est souvent appelée corrélateur $\beta$. Ce domaine présente en outre des propriétés d'échelle qui deviennent, en tenant compte de la relation (15),

$$
G(t) \propto \sqrt{\left|X-X_{e}\right|} g_{\lambda}\left(t / t_{\beta}\right),
$$

avec

$$
t_{\beta} \propto\left|X-X_{x}\right|^{-1 / 2 a}
$$

et $g_{\lambda}$ est une fonction d'échelle paramétrée uniquement par $\lambda$.

Quant à la seconde étape de la dynamique, elle correspond aux processus de relaxation les plus lents, c'est-à-dire à la relaxation $\alpha$. On déduit donc des équations (12) et (15) que la théorie prédit une divergence en loi de puissance du temps caractéristique de la relaxation $\alpha$

$$
\tau_{\alpha} \propto\left|X-X_{x}\right|^{-\gamma},
$$

avec $\gamma=\frac{1}{2 a}+\frac{1}{2 b}$ supérieur ou égal à 1.765 .

Pour finir, deux remarques s'imposent. D'abord, toutes les propriétés présentées dans ce chapitre sont des propriétés asymptotiques et n'incluent que les contributions à l'ordre dominant en $\sigma$. Elles ne sont donc valables que pour des temps suffisamment longs et dans un voisinage suffisamment restreint de la bifurcation sous peine dans le cas contraire d'être masquées ou altérées par des contributions dues à des corrections préasymptotiques. Ensuite, les propriétés évoquées ici ne sont démontrées en toute rigueur que pour les équations de la théorie appliquée aux liquides monoatomiques à un seul constituant. Elles paraissent néanmoins demeurer valables dans le cas des extensions de la théorie aux mélanges de liquides atomiques ou aux fluides moléculaires et il semble raisonnable de penser que si ce scénario fondé sur un certain type de rétroaction non linéaire est valable d'une quelconque manière il doit présenter un large degré d'universalité. On s'attend donc à ce que le phénomène de transition vitreuse idéale et l'ensemble des résultats spécifiques décrits dans ce chapitre s'appliquent de façon assez générale à la description de la dynamique lente de la plupart des liquides quelle que soit leur complexité chimique. 


\subsection{Tester la théorie de couplage de modes de la transition vitreuse}

La théorie de couplage de modes de la transition vitreuse fait nombre de prédictions détaillées susceptibles d'être testées expérimentalement ou par simulation numérique, tant d'un point de vue qualitatif, à travers les propriétés génériques universelles mises en évidence au chapitre précédent, que quantitatif, lorsque toutes les données structurales nécessaires à la résolution des équations telles que (3) sont disponibles. Elle a par conséquent suscité un grand nombre d'investigations portant sur de multiples systèmes et utilisant les techniques les plus diverses.

\subsubsection{Le scénario étendu}

Avant de présenter les différents tests de la théorie de couplage de modes de la transition vitreuse, il est utile de considérer certaines modifications du scénario présenté plus haut qui s'avèrent nécessaires pour créer le lien entre les prédictions théoriques et les observations expérimentales. La théorie de couplage de modes présentée à la section précédente, qui est dite idéale, permet de rendre compte d'un certain nombre de propriétés qualitatives de la dynamique des liquides surfondus, telles que l'augmentation rapide du temps de relaxation structurale ou le développement d'une relaxation en deux étapes, en relation avec la prédiction de l'existence d'une transition dynamique correspondant à une brisure d'ergodicité. Mais il est maintenant bien connu que ce scénario ne représente qu'imparfaitement la réalité. En effet, aussi bien les résultats expérimentaux que les développements théoriques conduisant à ce qui est couramment appelé une théorie étendue concourent à démontrer que la théorie idéale surestime la tendance qu'a un liquide à former un verre et que la transition vitreuse idéale est en fait évitée à cause de processus mal identifiés, appelés processus activés, qui n'ont pas été pris en compte comme voies possibles de décroissance des fluctuations des variables lentes retenues dans le calcul.

Le scénario de transition vitreuse idéale n'est donc pas observable tel quel et il est nécessaire de faire des hypothèses supplémentaires sur la façon dont il est susceptible de se manifester expérimentalement. L'hypothèse qui est couramment faite est celle d'une transition vitreuse idéale faiblement évitée, c'est-à-dire que les processus responsables de l'évitement de la transition sont suffisamment peu importants pour que l'essentiel des comportements prédits par la théorie subsistent malgré l'évitement de la transition. Le ralentissement de la dynamique est ainsi supposé bien décrit par l'approche idéale jusqu'à ce que, près de la transition, un changement de régime induit par les processus qui ont été négligés se produise. On considère alors qu'il existe une séparation d'échelles de temps telle que la théorie décrive bien les premiers temps de la relaxation, qui correspondent à la décroissance jusqu'au plateau, tandis que la décroissance à partir du plateau peut être exclusivement attribuée aux processus activés. En outre, dans la mesure où il n'y a pas de véritable brisure d'ergodicité observée expérimentalement là où elle est attendue, pour tout ce qui touche aux propriétés du paramètre de non-ergodicité, il est nécessaire de modifier la caractérisation de cette grandeur, qui sera généralement définie de façon opérationnelle comme l'amplitude de la relaxation $\alpha$ ou encore comme la valeur d'une fonction de corrélation au voisinage du plateau.

\subsubsection{Tests qualitatifs}

La plupart des tests de la théorie de couplage de modes de la transition vitreuse sont de nature qualitative, c'est-à-dire qu'ils visent à mettre en évidence les comportements universels 
prédits par la théorie et qui ont été présentés plus hauts. Il s'agit de la seule approche possible pour la plupart des liquides vitrifiables dans la mesure où ce sont souvent des systèmes moléculaires complexes inabordables de façon quantitative par la théorie. Mais ces techniques sont également intéressantes pour les systèmes qui supportent une étude quantitative, car ce sont souvent elles qui servent à extraire les grandeurs à comparer aux résultats théoriques. On peut essentiellement distinguer deux catégories de tests qualitatifs.

Le premier type d'approches qualitatives regroupe les méthodes qui se focalisent plutôt sur l'évolution globale de la dynamique et cherchent à caractériser les comportements singuliers prédits par la théorie idéale au point de transition vitreuse idéale. Ces comportements sont, d'une part, la divergence en loi de puissance des temps de relaxation $\alpha$, et, d'autre part, le comportement en racine carrée du facteur de non-ergodicité.

Les lois relatives aux temps de relaxation $\alpha$ et leurs corollaires relatifs aux coefficients de transport,

$$
\begin{aligned}
\text { viscosité: } & \eta \propto\left|X-X_{c}\right|^{-\gamma}, \\
\text { constante de diffusion : } & D \propto\left|X-X_{c}\right|^{\gamma},
\end{aligned}
$$

ne sont généralement observés que sur quelques ordres de grandeur, ce qui n'est pas suffisant pour être catégorique quant à leur validité (cf figure 14). Elles ne jouent donc qu'un rôle secondaire lorsqu'il s'agit de tester la théorie sur des systèmes réels. En revanche, en dépit de cette limitation, elles jouent un rôle important dans les travaux par simulation numérique, où elles sont largement utilisées pour déterminer la position de la transition vitreuse idéale à partir des données. Par ailleurs, elles permettent en principe un premier test de cohérence de la théorie qui prédit que les valeurs de $X_{c}$ et de l'exposant $\gamma$ sont les mêmes pour toutes les grandeurs.

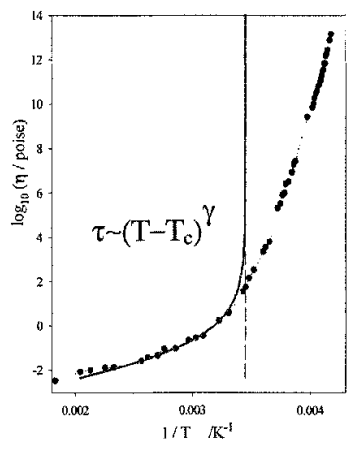

Figure 14: évolution de la viscosité de l'o-terphenyl et détermination de Tc

Les travaux expérimentaux impliquant la recherche de l'anomalie du paramètre de nonergodicité ont, en comparaison, une importance considérablement plus grande. Compte tenu de la définition opérationnelle de cette grandeur, qui a été donnée plus haut, le paramètre de nonergodicité n'est plus discontinu à la transition et la singularité attendue est donc de la forme :

$$
f_{G}-f_{G}^{c}=\left\{\begin{array}{lr}
\alpha_{G}\left(X-X_{c}\right)+o\left(X-X_{c}\right) & \text { (liquide), } \\
\mathrm{A}_{G} \sqrt{\left|X-X_{c}\right|}+\alpha_{G}\left(X-X_{c}\right)+o\left(X-X_{c}\right) & \text { (verre idéa), }
\end{array}\right.
$$

(l'indice $\mathrm{G}$ signale les paramètres qui dépendent de la grandeur mesurée). Schématiquement, deux méthodes sont essentiellement utilisées pour obtenir le paramètre de non-ergodicité, en fonction 
des caractéristiques de l'expérience. Si on considère une expérience dont l'échelle de temps caractéristique excède le temps de relaxation $\alpha$, il va être possible de mesurer la décroissance complète des fluctuations en fonction du temps. On extrait alors le paramètre de non-ergodicité, défini comme l'amplitude du processus $\alpha$, par ajustement numérique de la deuxième étape de la relaxation avec une fonction ad hoc adaptée, par exemple une exponentielle étirée. Cette méthode se transpose directement au cas d'une mesure en fréquence où c'est alors le pic $\alpha$ qui fait l'objet d'un ajustement numérique. Le paramètre de non-ergodicité peut également être obtenu dans le cadre d'une investigation du processus $\beta$ dont le principe sera décrit plus loin. Si en revanche l'échelle de temps de l'expérience est plus courte que le temps de relaxation $\alpha$, alors le système étudié répond à la définition opérationnelle de l'état vitreux et les fluctuations mesurées vont apparaître figées. On peut alors simplement prendre comme valeur du paramètre de nonergodicité la valeur de ces fluctuations figées. Si la mesure est faite en fréquence, cette situation correspond au cas où le pic $\alpha$ est à l'intérieur de la bande de fréquence autour de la fréquence nulle que l'expérience ne permet pas résoudre. La valeur de l'intensité "élastique" qui correspond à cette bande de fréquence s'identifie alors au paramètre de non-ergodicité.

Le second type d'approches qualitatives s'intéresse de façon plus détaillée à la forme de la dynamique en fonction du temps ou de la fréquence, pour vérifier la validité des propriétés relatives au processus $\beta$ (cf figure 15). On cherche alors à montrer qu'il est possible de décrire l'allure d'une fonction de corrélation au voisinage du plateau par une expression de la forme

$$
\phi_{G}(t)=f_{G}^{c}+A_{G, \beta} g_{\lambda}\left(t / t_{\beta}\right)
$$

ou l'allure d'une susceptibilité au voisinage de son minimum par la formule

$$
\chi_{G}{ }^{\prime \prime}(\omega)=A_{G, \beta} \omega g_{\lambda} "\left(\omega / \omega_{\beta}\right) \text {, }
$$

$g_{\lambda}$ étant une fonction d'échelle connue paramétrée par $\lambda$. Le calcul de $g_{\lambda}$ étant difficile, différentes formules approchées ont été proposées, dont les plus utilisées réalisent une simple interpolation entre les deux lois de puissance en $a$ et $b$ :

$$
\begin{gathered}
\phi_{G}(t)=f_{G}^{c}+A_{G, \beta}\left(b\left(\frac{t_{\beta}}{t}\right)^{a}-a\left(\frac{t}{t_{\beta}}\right)^{b}\right), \\
\chi_{G}^{\prime \prime}(\omega)=\frac{\chi_{G, \min }}{a+b}\left(b\left(\frac{\omega}{\omega_{\min }}\right)^{a}+a\left(\frac{\omega_{\min }}{\omega}\right)^{b}\right),
\end{gathered}
$$

où les exposants $a$ et $b$ sont contraints de vérifier la relation qui les lie au paramètre d'exposant $\lambda$. À ces formules d'ajustement s'ajoutent les propriétés d'échelle

$$
\begin{array}{ll}
t_{\beta} \propto\left|X-X_{x}\right|^{-1 / 2 a} & \text { indépendant de } \mathrm{G}, \\
\omega_{\beta}, \omega_{\min } \propto\left|X-X_{x}\right|^{1 / 2 a} & \text { indépendants de } \mathrm{G}, \\
A_{G, \beta}, \chi_{G, \min } \propto \sqrt{\left|X-X_{x}\right|}, &
\end{array}
$$

qui permettent de déterminer la position de la transition vitreuse idéale et auxquelles on peut d'ailleurs adjoindre celle qui s'applique aux temps de relaxation $\alpha$, car la valeur de l'exposant $\gamma$ est reliée de façon univoque à la valeur de $\lambda$.

Toutes ces approches permettent de déterminer la position de la transition vitreuse idéale prédite par la théorie. Comme cela a été anticipé à la section précédente, les résultats expérimentaux montrent sans ambiguïté que, si une transition vitreuse idéale est d'une 
quelconque manière à l'origine du ralentissement de la dynamique des liquides surfondus, celle-ci est en réalité évitée et devrait, si elle se produisait vraiment et si des processus additionnels ne venaient pas restaurer l'ergodicité à basse température et haute densité, être localisée bien audessus de la température de transition vitreuse calorimétrique, dans un domaine où la relaxation structurale a un temps caractéristique de l'ordre de 10-100 ns.

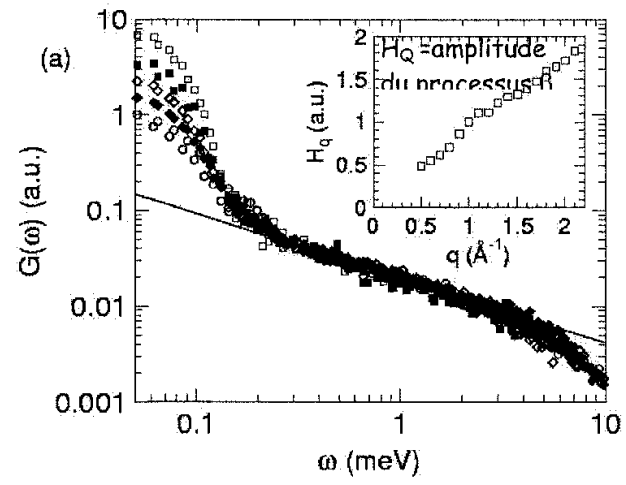

Figure 15: test de factorisation du facteur de structure incohérent $\mathrm{du}$ toluène à $145 \mathrm{~K}, \mathrm{~S}(\mathrm{Q}, \mathrm{w}) / \mathrm{S}(\mathrm{Q}) \mathrm{H}_{\mathrm{Q}}$ pour $\mathrm{Q}=.5 \mathrm{a} 2 \AA^{-1}$. On obtient une courbe maitresse du processus $\beta$ avec une loi de puisssance $\omega^{\mathrm{a}-\mathrm{l}}$, où $\mathrm{a}=33 ; \mathrm{H}_{\mathrm{Q}}$ est proportionnel à l'amplitude de la relaxation $\beta$.

\subsubsection{Tests quantitatifs}

Pour aller au-delà des approches ci-dessus, qui permettent de mettre en évidence certaines des propriétés génériques prédites par la théorie de couplage de modes au voisinage d'une transition vitreuse idéale, la question se pose de savoir si, en plus d'un accord qualitatif, la théorie est également capable d'être quantitative, c'est-à-dire de prédire pour un système donné des grandeurs non universelles comme la température de transition ou le paramètre de non-ergodicité en fonction du vecteur d'onde à la transition. Une telle étude est en principe possible sur tous les systèmes pour lesquels la théorie a été complètement développée à partir des premiers principes, tels que les liquides atomiques et leurs mélanges, en comparant directement pour un système donné la dynamique telle qu'elle est prédite par la théorie aux résultats obtenus par l'expérience ou les simulations. Le seul pré-requis est alors la connaissance avec une précision suffisante des propriétés structurales du liquide, qui peuvent être déduites de l'expérience ou de la simulation, ou obtenues par des méthodes théoriques (équations intégrales par exemple).

Il n'existe à ce jour qu'un seul système expérimental ayant été étudié suivant ces modalités. Il s'agit d'une suspension colloïdale de particules sphériques qui peut être représentée de façon satisfaisante par un liquide de sphères dures et dont la dynamique des fluctuations de densité étudiée par diffusion de la lumière a été comparée de façon extensive aux résultats théoriques obtenus pour le liquide modèle correspondant.

Les autres études quantitatives ont été effectuées par simulation numérique. En effet, diverses restrictions rendent l'approche expérimentale malaisée. D'abord, les systèmes monoatomiques et les liquides moléculaires les plus simples cristallisent très facilement et sont dans la pratique impossibles à étudier expérimentalement dans l'état liquide surfondu. Ensuite, il est difficile d'obtenir l'information structurale requise pour résoudre les équations de la théorie. Enfin, même si la dynamique prédite par la théorie était calculée, la connexion entre celle-ci et les résultats expérimentaux disponibles demeurerait difficile à établir. Tous ces problèmes trouvent une solution dans le recours à la simulation numérique. Celle-ci permet de limiter les 
problèmes posés par la cristallisation grâce à des vitesses de trempe très élevées et à la possibilité de choisir un potentiel d'interaction qui défavorise la formation du cristal. En outre, il est possible de déterminer les corrélations statiques nécessaires à la théorie et d'obtenir directement les grandeurs dynamiques pour lesquelles elle fait des prédictions, rendant ainsi immédiatement réalisable la comparaison théorie/expérience numérique. Ces avantages concourent à faire de la simulation numérique une méthode de choix pour les tests quantitatifs de la théorie de couplage de modes de la transition vitreuse.

Le tout premier élément de comparaison entre théorie et expériences ou simulations porte évidemment sur la position d'une éventuelle transition vitreuse idéale. La plupart du temps, celleci est identifiée à partir des données en utilisant l'une ou l'autre des méthodes décrites à la section précédente (on peut noter que, pour les simulations, la méthode fondée sur la singularité du paramètre de non-ergodicité est généralement inopérante, car il s'avère difficile d'obtenir des simulations à l'équilibre au voisinage et au-delà du point de transition vitreuse idéale, qui est donc déterminé par extrapolation). On trouve alors de façon générale que le résultat théorique surestime le ralentissement de la relaxation structurale, c'est-à-dire que typiquement la densité de transition calculée est inférieure de $10 \%$ à celle qui peut être déduite de l'expérience ou de la simulation, une incertitude qui, dans le cas d'une étude isochore, peut se traduire par une erreur d'un facteur 2 sur la température de transition (Nauroth et Kob 1997).

Cette erreur sur la localisation de la transition vitreuse idéale est susceptible de se reporter sur les valeurs de toutes les autres grandeurs prédites par la théorie. Un accord quantitatif entre théorie et données expérimentales ou de simulation pour ces grandeurs n'est donc pas évident $a$ priori. Diverses études plus détaillées des prédictions de la théorie ont néanmoins été proposées.

Nombre d'entre elles portent sur la dépendance du facteur de Debye-Waller en fonction du vecteur d'onde (et des indices orientationnels dans le cas des systèèmes moléculaires). On compare alors les valeurs prédites par la théorie au point de transition calculé à celles qui sont obtenues par l'une quelconque des méthodes décrites précédemment au voisinage du point de transition estimé à partir de la dynamique. En général, seul un accord qualitatif est obtenu, c'està-dire que le paramètre de non-ergodicité oscille en phase avec la grandeur structurale à laquelle il est directement relié (fonction de distribution de paire ou facteur de structure statique selon qu'on travaille dans l'espace réel ou réciproque), en accord avec les prédictions théoriques, (cf figure 16).

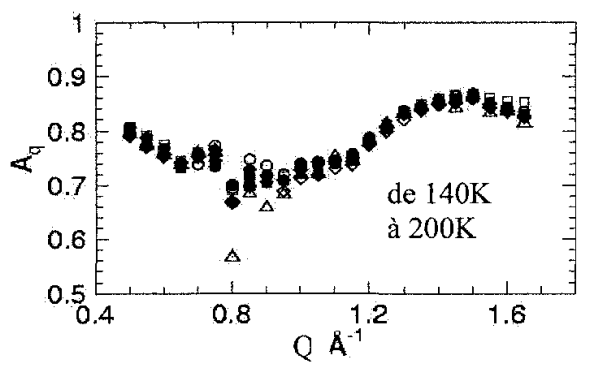

Figure 16: variation du facteur de Debye Waller du toluène calculé à partir d'un ajustement de la loi de Kohlraush sur des mesures cohérentes de la relaxation par echo de spin.

Il est également possible, à partir du seul calcul des résultats à temps infini, de déterminer la valeur théorique du paramètre d'exposant $\lambda$ pour le système étudié. La valeur de $\lambda$ étant difficile à extraire de façon univoque des données expérimentales ou de simulation, ce sont surtout des tests de compatibilité qui ont été réalisés, c'est-à-dire qu'on vérifie qu'il est possible de 
rendre compte de la dynamique du système étudié grâce aux exposants ou à la forme $d u$ processus $\beta$ qui correspondent à la valeur de $\lambda$ calculée.

À ce jour peu d'études quantitatives sont allées plus loin dans la comparaison entre la théorie et l'expérience ou la simulation, en s'intéressant à l'ensemble de la dynamique lente, et non plus seulement aux paramètres de non-ergodicité et aux prédictions génériques de la théorie qui ne nécessitent qu'un calcul de grandeurs indépendantes du temps. Une telle étude a été entreprise dans le cas de la suspension colloïdale étudiée par diffusion de la lumière. Enfin, on peut mentionner le travail de Nauroth (1999) qui propose un calcul complet de la dynamique d'un mélange binaire de Lennard-Jones qui inclue non seulement les effets de couplage de modes qui conduisent à la transition vitreuse idéale, mais aussi un traitement soigné de la dynamique rapide. Il est alors possible de comparer théorie et simulations de façon extensive sur toute l'étendue de la dynamique.

\subsubsection{Limites et désaccords}

Il existe de nombreux résultats qui montre un bon accord avec les prédictions théoriques et plaident donc fortement en faveur de la théorie de couplage de modes. Mais il en existe aussi un nombre conséquent qui contredisent la théorie ou du moins ne présentent pas les caractéristiques attendues. C'est par exemple le cas des données obtenues pour le CKN par Mezei et Russina (1999) pour lesquelles aucune singularité du paramètre de non-ergodicité n'est visible. Par ailleurs, il est souvent difficile de déterminer sans ambiguïté des paramètres importants de la théorie, comme le montre le travail de Aouadi et al. (2000) où on voit que le paramètre de nonergodicité extrait de spectres de diffusion Brillouin dépend fortement du modèle phénoménologique utilisé pour sa détermination.

En fait, un certain nombre de questions se posent quant aux modalités selon lesquelles la théorie est testée expérimentalement. Elles sont particulièrement aiguës dans le cas des investigations qui portent sur le détail de la dynamique, auxquelles nous limitons la discussion qui suit.

D'abord la théorie de couplage de modes idéale est une théorie de la relaxation structurale et ne fait donc aucune prédiction sur la dynamique aux temps courts. Celle-ci est d'ailleurs généralement prise en compte de façon très grossière à travers une approximation markovienne. Il est donc nécessaire pour pouvoir tester la théorie que les deux types de dynamique se produisent sur des échelles de temps bien séparées. Or il n'est pas évident a priori que cette condition puisse être réalisée. En effet, alors que la théorie prédit cette séparation des échelles de temps à travers la divergence des temps caractéristiques des processus $\alpha$ et $\beta$, nous savons que des voies de relaxation qui n'ont pas été prises en compte conduisent à l'évitement de la transition vitreuse idéale et limitent l'augmentation des temps de relaxation qui demeurent finis. Il en résulte que le domaine de validité éventuelle de la théorie idéale est limité à des temps de relaxation $\alpha$ modérés, au-delà desquels la dynamique est dominée par d'autres effets que ceux de couplage de modes. La question est alors de savoir si la séparation des échelles de temps est alors suffisante, ce qui ne semble pas être toujours le cas.

Un deuxième aspect du problème qui s'ajoute au précédent est la question de l'observabilité des prédictions asymptotiques de la théorie, qui ne sont valables quau voisinage de la transition vitreuse idéale et qui sont au cœur de la plupart des tests de la théorie. Ce n'est que récemment que les corrections préasymptotiques à ces prédictions et leur domaine de validité ont pu être étudiés en détail pour un fluide modèle de sphères dures traitées dans l'approximation de Percus- 
Yevick (Franosch et al. (1997), Fuchs et al. (1998). Il s'avère que les propriétés asymptotiques ne sont valables que très près de la transition, lorsque plus de 4 décades de temps séparent la dynamique microscopique de la relaxation $\alpha$. Or cette limite coüncide approximativement avec celle du domaine de validité éventuelle de la théorie idéale. Il n'est donc pas certain que le régime asymptotique ait pu se développer suffisamment pour être identifiable dans le domaine d'étude considéré.

Enfin, d'un point de vue totalement différent, on peut envisager une remarque de pure forme. Il semble regrettable de tester la théorie en se concentrant sur l'examen d'un domaine limité de la dynamique (plateaux de fonctions de corrélation ou minima de susceptibilités) et en excluant une large part de l'information disponible; il est apparu alors souhaitable de disposer de méthodes pour tester la théorie de couplage de modes qui ne soient pas restreintes aux prédictions asymptotiques de la théorie et qui puissent rendre compte de l'ensemble de la dynamique en prenant en considération le maximum de données expérimentales disponibles. Idéalement, de telles méthodes permettraient un examen critique des deux objections soulevées ici relatives à la séparation des échelles de temps et à l'existence d'un régime asymptotique. C'est précisément ce qu'il est possible de faire en utilisant des modèles phénoménologiques issus de la théorie de couplage de modes appelés modèles schématiques (Krakoviack et al. 2002).

\section{CONCLUSION}

L'étude par diffusion quasiélastique de neutrons de la dynamique de liquides visqueux a permis d'identifier clairement la forme, la dépendance en température et en vecteur d'ondes du processus principal de relaxation dit $\alpha$ ou structurale lorsque le système approche la transition vitreuse. Des propriétés universelles ont été déduites à partir d'un grand nombre d'espèces chimiques différentes mettant en jeu des intéractions intermoléculaires distinctes (de liquides simples, à liaison hydrogène, ioniques ou polymèriques): étirement de la relaxation, développement en deux étapes, processus rapide aux temps courts. La complémentarité très favorable des expériences et des simulations numériques ont permis d'éclaircir et d'approfondir de nombreux points, en particulier sur la corrélation des mouvements de rotation et de translation.

L'apparition de la théorie dite de couplage de modes a largement stimulé et contribué au développement de ces études expérimentales et numériques dans le domaine temporel de la nanoseconde ; certaines prédictions ont été vérifiées avec plus ou moins de succès dans les barres d'erreur experimentales, mais aucun comportement critique à proprement parlé n'a pu être clairement mis à jour ni totalement écarté.

L'étude du phénomène de vitrification demande par ailleurs la combinaison de plusieurs techniques expérimentales telles que la diffusion de la lumière, la spectroscpie diélectrique ou la RMN. Il existe aussi d'autres approches théoriques prenant en compte sur l'augmentation spectaculaire des temps de relaxations à l'approche de la température de gel $\mathrm{Tg}$, la notion de fragilité développée par Angell, ainsi que le caractère non-exponentielle de la relaxation structurale en fonction du temps ou la présence d'hétérogénéités dynamiques. Citons la théorie récente des domaines limités par la frustration (Kivelson et al 1994), qui propose de décrire les divers changements dans la dynamique des liquides surfondus comme la conséquence d'un phénomène universel de frustration et aussi l'approche phénoménologique d'Adam et Gibbs (1965), qui corrèle l'accroissement du temps de relaxation à la décroissance de l'entropie. Les 
méthodes d'analyse appliquées dans ce cadre d'étude peuvent être étendues à des conditionnements de fluides variés, tels que des effets de compression ou de confinement, mais aussi à un domaine de recherches beaucoup plus large dès lors qu'un processus de relaxation complexe apparaît ; citons dans ce cas l'étude de la dynamique rapide des proteines.

\section{Références}

Brawer S.A., J. Chem. Phys. 81, 954 (1984)

Vogel H., Z. Phys. 22, 645 (1921) ; G.S. Fulcher J. Am. Ceram. Soc. 8, 339, 1925 ; G. Tammann, W.Z. Hesse, Anorg. Allgem. Chem. 156, 245 (1926).

Angell C.A.(1985) in Relaxations in complex systems, ed. K.Ngai and G.B.Wright, N.T.I.S., U.S. department of Commerce, Springfield, VA 22161, p1.

Kauzmann W., Chem. Rev. 43, 219 (1948).

Debye P., Phys.Z., 3, 97-100, (1912); Polar Molecules (New York, Dover, 1929)

Kohlrausch R., Pogg. Ann. Phys. 12(3), (1854) 393 ; ibid V(8) (1866) 1, 207, 399

Williams G., Watts D.C., Trans.Faraday Soc. 66, 80-85,(1970).

Davidson D.W., Cole R.H., J. Chem. Phys. 19, (1951) 1484

Sillescu H., J. Non-Cryst. Solids, 243 (1999) 81-108

Johari G.P., Golstein M., J. Chem. Phys. 53, 2372-2388 (1970)

Arbe A., Richter D., Colmenero J., Farago B., Phys.Rev. E, 54, 3853 (1996)

Lunkenheimer P., Schneider U., Brand R., Loidl A., Cont. Phys. 41,(2000), 15.

Morineau D., Alba-Simionesco, J. Chem. Phys. 109, 8494 (1998).

Gomez D., A. Alegria, J. Non-Cryst. Solids 287 (2001) 246-251

Van Hove L., Phys. Rev., 95, 249 (1954).

de Gennes P.-G., Physica, 25, 825 (1959).

Sköld K., Rowe J.M.,Ostrowski G.,Randoph P.D.,Phys. Rev. A 6, 1107 (1972).

C. Alba-Simionesco, A. Tölle, D. Morineau, B. Farago, G. Coddens, cond-mat/0103599 (2000);

A. Tölle, C.Alba-Simionesco, J. Chem.Phys., sous presse 2003. 
Kob W. and Andersen H. C., Phys. Rev. E 51, 4626 (1995). Wahnstro"m G. and Lewis L. J., Physica A 201, 150 (1993); Mossa S.,et al.Phys. Rev. E 62, 612 (2000); Teboul V., AlbaSimionesco C. in preparation.

Mezei F.in "Liquids, Freezing, and the Glass Transition", J. P. Hansen, D. Levesque, and J. Zinn-Justin Eds. (North Holland, Amsterdam, 1991), pg. 629.

Schärpf O., Capellmann H., Phys. Satus Solidi A, 135, 359 (1993).

Tölle A.,Rep. Prog. Phys. 64,1473-1532 (2001).

Frick B., Alba-Simionesco C., J. Applied Physics A 2002.

Arbe A., Colmenero J., Monkenbusch M., et Richter D., Phys. Rev. Lett. 81 (3), 590(1998). A. Arbe, A. Alegría, F. Alvarez, J. Colmenero, and B. Frick, Suppl. Colloid and Polym. Sci. 270 (1992). B. Farago, A. Arbe, J. Colmenero, R. Faust, U. Buchenau, and D. Richter, Phys. Rev. E65, 051803 (2002). J. Colmenero, F. Alvarez, and A. Arbe, Phys. Rev. E 65, 041808 (2002).

Kivelson D., Kivelson S.A., Zhao L., Nussimov Z., and Tarjus G., Physica A 219, 27 (1995). Kivelson, D., and Tarjus, G., Philos. Mag. B 77, 245 (1998).

Adam, G., and Gibbs, J. H., J. Chem. Phys. 43, 139 (1965).

Aouadi A., Dreyfus C., Massot M., Pick R.M., Berger T., Steffen W., Patkovski A. et AlbaSimionesco C., Light scattering study of the liquid glass transition of meta-toluidine, J. Chem. Phys., (2000).

Franosch T., Fuchs M., Götze W., Mayr M. et Singh A., Asymptotic laws and preasymptotic correction formulas for the relaxation near glass-transition singularities, Phys. Rev. E 55, 7153 (1997).

Fuchs M., Götze W., Hildebrand S. et Latz A., $\beta$ relaxation theory for simple systems: some addenda, Zeit. Phys. B 87, 43 (1992).

Fuchs M., Götze W. et Mayr M., Asymptotic laws for tagged-particule motion in glassy systems, Phys. Rev. E 58, 3384 (1998).

Geszti T., Pre-vitrification by viscosity feedback, J. Phys. C: Solid State Phys. 16, 5805 (1983).

Mezei F. et Russina M., Intermediate range order dynamics near the glass transition, J. Phys.: Condens. Matter 11, A341 (1999).

Nauroth M. et Kob W., Quantitative test of the mode-coupling theory of the ideal glass transition for a binary Lennard-Jones system, Phys. Rev. E 55, 657 (1997). 
Nauroth M.H., Die Dynamik von normalen und unterkühlten Flüssigkeiten. Ein Vergleich von analytischen Theorien mit Computersimulationen, thèse de l'université technique de Munich, 1999.

van Megen W. et Underwood S.M., Glass transition in colloidal hard spheres: mode-coupling theory analysis, Phys. Rev. Lett. 70, 2766 (1993).

van Megen W. et Underwood S.M., Glass transition in colloidal hard spheres: measurement and mode-coupling-theory analysis of the coherent intermediate scattering function, Phys. Rev. E 49, 4206 (1994).

Krakoviack V. et Alba-Simionesco C. J.Chem.Phys. 117(2002), 2161; Physics of glasses, structure and dynamics, AIPconference 489, 243(1999)

Quelques revues sur le phénomène de la transition vitreuse:

J. Jackle, Rep. Prog. Phys. 49, 171 (1986), C. A. Angell, Science 267, 1924 (1995) ; G. Tarjus, D. Kivelson, S. Kivelson, in Supercooled Liquids: Advanced and Novel Application, J. Fourkas, et al eds., American Chemical Society, Baltimore,1997 ; M. D. Ediger, C. A. Angell, and S. Nagel, J. Phys. Chem. 100, 13200 (1996) ; K. L. Ngai, J. of Non-Cryst. 275,(2000),7-51 ; Proceedings of the International Meetings on "Relaxation in Complex Systems": (i) K. L. Ngai and G. B. Wright Eds., Office of Naval Research, Arlington (1985); (ii) K. L. Ngai and G. B. Wright Eds., J. Non-Cryst. Solids 131-133 (1991); (iii) K. L. Ngai, E. Riande, and G. B. Wright Eds., J. Non-Cryst. Solids 172-174 (1994); (iv) K. L. Ngai Ed., J. Non-Cryst. Solids 235-238 (1998); Physics of glasses, structure and dynamics, ed. P.Jund and R.Jullien, AIPconference 489, 243(1999); P.G. Debenedetti, Metastable liquids. Concepts and Principles. Princeton university Press, 1996. Edition spéciale des Comptes Rendus de l'Académie des Sciences, Serie IV, 2001. 